Y. Ikeda

Nagoya Math. J.

Vol. 94 (1984), 105-135

\title{
WEAK SOLUTIONS OF A QUASI-LINEAR DEGENERATE ELLIPTIC SYSTEM WITH DISCONTINUOUS COEFFICIENTS
}

\author{
YOSHIAKI IKEDA
}

\section{§`1. Introduction}

We shall discuss regularities and related topics on weak solutions of the system of the following quasi-linear elliptic differential equations (a combination of almost single equations)

$$
\begin{aligned}
& -\operatorname{div} A_{j}\left(x, u, \nabla u_{j}\right)+B_{j}\left(x, u, \nabla u_{j}\right)=0 \\
& (j=1,2, \cdots, m) \quad u=\left(u_{1}, \cdots, u_{m}\right),
\end{aligned}
$$

in a bounded domain $\Omega$ in $R^{n}(n \geqq 2)$, where $A_{j}=\left(A_{1 j}, \cdots, A_{n j}\right)$ are given vector functions of $\left(x, u, \nabla u_{j}\right), B_{j}$ are scalar functions of the same variables, and $\nabla u_{j}=\left(\partial u_{j} / \partial x_{1}, \cdots, \partial u_{j} / \partial x_{n}\right)$ denote the gradients of the $u_{j}=u_{j}(x)$ $(j=1, \cdots, m)$. We assume that there exists some $\alpha \geqq 2$ such that each $A_{j}$ and $B_{j}$ satisfy the inequalities

$$
\left\{\begin{array}{l}
\xi \cdot A_{j}(x, u, \xi) \geqq a_{j}(x)|\xi|^{\alpha}-\sum_{i=1}^{m} c_{i j}(x)\left|u_{i}\right|^{\alpha}-f_{j}(x), \\
\left|B_{j}(x, u, \xi)\right| \leqq b_{j}(x)|\xi|^{\alpha-1}+\sum_{i=1}^{m} d_{i j}(x)\left|u_{i}\right|^{\alpha-1}+g_{j}(x), \\
\left|A_{j}(x, u, \xi)\right| \leqq \tilde{a}_{j}(x)|\xi|^{\alpha-1}+\sum_{i=1}^{m} e_{i_{j}}(x)\left|u_{i}\right|^{\alpha-1}+h_{j}(x),
\end{array}\right.
$$

for any $\xi \in R^{n}$. The functions $a_{j}, b_{j}, c_{i j}, \cdots, h_{j}$ and $\tilde{a}_{j}$, call them the coefficients of the structure (1.2), are all assumed to be non-negative and measurable.

Moreover we assume that

$$
\begin{aligned}
& a_{j}^{-1} \in L^{t}(\Omega) \quad \text { for any } t>1, \\
& a_{j} \leqq \tilde{a}_{3}, \quad j=1,2, \cdots, m,
\end{aligned}
$$

Received February 22, 1983. 


$$
\begin{aligned}
& \tilde{a}_{j}, \tilde{a}_{j}^{\alpha} a_{j}^{1-\alpha}, b_{j}^{\alpha} a_{j}^{1-\alpha}, c_{i j}, d_{i j}, f_{j}, g_{j} \in L^{p / \alpha}(\Omega), \\
& e_{i j}^{\alpha /(\alpha-1)} \tilde{a}_{j}^{1 /(1-\alpha)}, h_{j}^{\alpha /(\alpha-1)} \tilde{a}_{j}^{1 /(1-\alpha)} \in L^{p / \alpha}(\Omega)
\end{aligned}
$$

where

$$
\frac{\alpha}{p}+\frac{1}{t}<\frac{\alpha}{n} \text { and } \quad(2 \leqq) \alpha<p
$$

The class of Partial Differential Equations (1.1) that we are going to discuss involves many interesting equations whose solutions are known ([7, 10]). The purpose of this paper is to establish a systematic approach to the investigation of the solution, which may be weak solutions, of general equations in the class in question. Namely we shall discuss the topics 1) maximum principle 2) local boundedness 3) Hölder continuity 4) Harnack type inequality for the solutions.

We shall prove, under very general assumptions described above, the following theorems.

Theorem A. Let $u=\left(u_{1}, \cdots, u_{m}\right)$ be a weak solution of (1.1) such that $u=M$ on the boundary $\partial \Omega$ of $\Omega$ with $M=\left(M_{1}, \cdots, M_{m}\right) \in R^{m}$, then it holds that

$$
\sup _{\Omega}\left|u_{j}\right| \leqq C \sum_{i=1}^{m}\left\{\left(\int_{\Omega}\left|u_{i}\right|^{\alpha} d x\right)^{1 / \alpha}+\left(\int_{\Omega} f_{i}^{p / \alpha} d x\right)^{1 / p}+\left(\int_{\Omega} g_{\imath}^{p / \alpha} d x\right)^{\alpha /(\alpha-1) p}+\left|M_{i}\right|\right\}
$$

where $C$ is a positive constant depending only on $n, p, t, \alpha$ and the coeffcients of the structure (1.2).

Theorem B. Let $u=\left(u_{1}, \cdots, u_{m}\right)$ be a weak solution of (1.1) and $I\left(x_{0}, \rho\right)$ an open ball with radius $\rho$ and center at $x$. If $I\left(x_{0} .2 \rho_{0}\right) \subset \Omega$, then

$$
\sup _{I\left(x_{0}, \rho\right)}\left|u_{j}\right| \leqq C \sum_{i=i}^{m}\left\{\left(\rho_{0}^{-n} \int_{I\left(x_{0}, \rho_{0}\right)}\left|u_{i}\right|^{\alpha p /(p-\alpha)} d x\right)^{(p-\alpha) / \alpha p}+\rho_{0}^{n(p-\alpha) / \alpha p} \kappa_{i}\right\},
$$

where

$$
\begin{aligned}
\kappa_{i}=\left(\int_{I\left(2 \rho_{0}\right)} f_{i}^{p / \alpha} d x\right)^{1 / p} & +\left(\int_{I\left(2 \rho_{0}\right)} g_{i}^{p / \alpha} d x\right)^{\alpha /(\alpha-1) p} \\
& +\left(\int_{I\left(2 \rho_{0}\right)}\left(h_{i}^{\alpha /(\alpha-1)} a_{i}^{1 /(1-\alpha)}\right)^{p / \alpha} d x\right)^{\alpha /(\alpha-1) p} .
\end{aligned}
$$

We then proceed to prove the Hölder continuity for the weak solutions of (1.1) under the additional assumption that $\tilde{a}_{j} a_{j}^{-1}$ are bounded.

Theorem C. The weak solutions of (1.1) are locally Hölder contiunous in $\Omega$. 
If a solution is constant on $\partial \Omega$, then it is globally Hölder continuous in $\Omega$.

The Harnack type inequality can be proved under additional assumptions on the coefficients that are prescribed in Section 6 .

We now briefly summarise the contents of each sections. Section 2 is devoted to state some lemmas which will often be used later. We shall prove the maximum principle (Theorem A) and the theorems on local boundedness (Theorem B) in Section 3 and Section 4, respectively. By using these results we shall prove in Section 5 the Hölder continuity (Theorem C). The Harnack type inequality for positive weak solutions will be obtained in Section 6 . In the proofs of these results the techniques in Moser [4] and Stampacchia [8,9] are often used.

We now pause to give some historical notes on the development of the works in this line.

J. Moser [4] and G. Stampacchia [8] first proved all these properties for linear elliptic equations of the form

$$
-\sum_{i, j=1}^{n}\left(a_{i j}(x) u_{x_{i}}\right)_{x_{j}}=\sum_{i=1}^{n}\left(f_{i}\right)_{x_{i}} .
$$

Then, G. Stampacchia [9] extended these results for the strictly elliptic equations of the form

$$
-\sum_{i, j=1}^{n}\left(a_{i j}(x) u_{x_{i}}+d_{i} u\right)_{x_{j}}+\sum_{i=1}^{n}\left(b_{\imath}(x) u_{x_{i}}+c(x) u\right)=\sum_{i=1}^{n}\left(f_{i}\right)_{x_{i}},
$$

which are still linear.

While, J. Serrin [7] and N.S. Trudinger [10] proved the same results for weak solutions of a quasi-linear elliptic equations. These results are particular cases of our theorems, where $m=1$ and $a=a_{j}=$ constant in (1.1).

Another developments were made by M.K.V. Murthy and G. Stampacchia [5] and N.S. Trudinger [11] for the linear elliptic equations (1.4) in the case where the coefficients may be degenerated. F. Mandras $[1,2,3]$ considered the same problem in the case of a linear degenerate elliptic system.

Our results are actually viewed as a generalization of the above works, and our assumptions seems to be very general in order to prove the maximum principle, local boundedness and Hölder continuity. 
The author is grateful to Professor Tadato Matsuzawa for his many valuable suggestions to the author during the preparation of this paper.

\section{§ 2. Preliminaries}

In this section we shall state and prove several lemmas related to the imbedding theorems.

First of all, we shall define some function spaces; let $m(x)$ be a nonnegative measurable function in $\Omega$ and $m^{-1} \in L^{t}(\Omega)$ with $\alpha>1+1 / t, t>1$. The space $H^{1, \alpha}(m, \Omega)$ and $H_{0}^{1, \alpha}(m, \Omega)$ are the completions of $C^{\infty}(\Omega)$ and $C_{0}^{\infty}(\Omega)$ with the norm

$$
\|v\|_{H^{1, \alpha}(m, \Omega)}^{\alpha}=\int_{\Omega} m|\nabla v|^{\alpha} d x+\int_{\Omega}|v|^{\alpha} d x
$$

respectively. The Sobolev spaces appear as particular cases: $H^{1, \alpha}(1, \Omega)$ $=H^{1, \alpha}(\Omega)$ and $H_{0}^{1, \alpha}(1, \Omega)=H_{0}^{1, \alpha}(\Omega)$.

Throughout this paper, we denote by $\|f\|_{p}$ the $L^{p}(\Omega)$-norm.

Lemma 2.1 ([6]). Let $n \geqq 2$. If $v \in H_{0}^{1, p}(\Omega)(1<p)$, then

$$
\|v\|_{p *} \leqq c_{0}\|\nabla v\|_{p} .
$$

Here $1 / p^{*}=1 / p-1 / n$ if $p<n$, and $p^{*}$ may be taken to be any positive number if $p \geqq n$. The constant $c_{0}$ depends on $n$ and $p^{*}$. (If $p<n, c_{0}$ depends only on $n$.)

LEMmA 2.2. Let $n \geqq 2$. If $v \in H_{0}^{1, \alpha}(m, \Omega)$, then $v \in L^{\alpha^{\sharp}}(\Omega)$ and the following inequality holds

$$
\|v\|_{\alpha \sharp}^{\alpha} \leqq c_{0}\left\|m^{-1}\right\|_{t} \int_{\Omega} m|\nabla v|^{\alpha} d x .
$$

Here $1 / \alpha^{\#}=(1 / \alpha)(1+1 / t)-1 / n$ if $(1 / \alpha)(1+1 / t)>1 / n$, and $\alpha^{\#}$ may be taken to be any positive number $>1$ if $(1 / \alpha)(1+1 / t) \leqq 1 / n$. The constant $c_{0}$ is the same as in Lemma 2.1 .

Proof. Let $(1 / \alpha)(1+1 / t)>1 / n$. Then putting $1 / p=(1 / \alpha)(1+1 / t)$, we have $1 / p^{*}=1 / \alpha^{\sharp}$. Since $v \in H_{0}^{1, p}(\Omega)$, by Lemma 2.1 and Hölder's inequality, we have

$$
\|v\|_{a \#}^{\alpha} \leqq c_{0}\|\nabla v\|_{p}^{\alpha} \leqq c_{0}\left\|m^{-1}\right\|_{t} \int_{\Omega} m|\nabla v|^{\alpha} d x
$$

Next let $1 / \alpha(1+1 / t) \leqq 1 / n$. Then for any positive number $\alpha^{*}>1$, we 
take $\beta$ satisfying the equality $1 / \alpha^{\sharp}=(1 / \beta)(1+1 / t)-1 / n$. Since $1<\beta<\alpha$, using Lemma 2.1 and Hölder's inequality, we see

$$
\begin{aligned}
\|v\|_{\alpha \#}^{\alpha} & \leqq c_{0}^{1 / \beta}\left(\int_{\Omega}|\nabla v|^{\beta t /(t+1)} d x\right)^{\alpha(t+1) / \beta t} \\
& \leqq C\left(\int_{\Omega}|\nabla v|^{\alpha t /(t+1)} d x\right)^{(t+1) / t} \leqq C\left\|m^{-1}\right\|_{t} \int_{\Omega} m|\nabla v|^{\alpha} d x \text {. Q.E.D. }
\end{aligned}
$$

The following lemma is easily obtained by using Hölder's inequality.

LEMma 2.3. Lei $r$ and $s$ be such that $1 \leqq r \leqq s$. If $v \in L^{s}(\Omega)$, then for any $p$ with

$$
\frac{\lambda}{s}+\frac{\mu}{r}=\frac{1}{p} \quad(\lambda, \mu>0, \lambda+\mu=1)
$$

we have

$$
\|v\|_{p} \leqq\|v\|_{s}^{\lambda}\|v\|_{r}^{\mu}
$$

LEMMA 2.4. If $v \in H_{0}^{1, \alpha}(m, \Omega)$, then $v \in L^{\alpha p /(p-\alpha)}(\Omega)$ for any $p>0$ with $\alpha / n>1 / t+\alpha / p$. Moreover, for any positive number $\varepsilon$, there exists a constant $K$ depending only on $n, p, t, \alpha, \varepsilon$ and $\left\|m^{-1}\right\|_{t}$ satisfying the following inequality

$$
\|v\|_{\alpha p /(p-\alpha)}^{\alpha} \leqq \varepsilon \int_{\Omega} m|\nabla v|^{\alpha} d x+K \int_{\Omega}|v|^{\alpha} d x
$$

Here we may take

$$
K=\left(c_{0}\left\|m^{-1}\right\|_{t} \varepsilon\right)^{-\gamma}, \gamma=\frac{1-\lambda}{\lambda} \text { with } \lambda=\frac{1}{p} /\left(\frac{1}{\alpha}-\frac{1}{\alpha^{\sharp}}\right)<1 .
$$

Proof. Let $r$ and $s$ be real numbers $\geqq 1$. For $\lambda=\lambda(r, s)$ such that $0<\lambda<1$ and $\lambda / s+(1-\lambda) / r=(p-\alpha) /(\alpha p)$. We have by Lemma 2.3 and Young's inequality

$$
\|v\|_{\alpha p /(p-\alpha)}^{\alpha} \leqq\|v\|_{s}^{\alpha \lambda}\|v\|_{r}^{\alpha(1-\lambda)} \leqq \lambda \varepsilon^{\prime}\|v\|_{s}^{\alpha}+(1-\lambda) K^{\prime}\|v\|_{r}^{\alpha}, \quad K^{\prime}=\varepsilon^{\prime-\lambda /(1-\lambda)}
$$

for any $\varepsilon^{\prime}>0$.

If $(1 / \alpha)(1+1 / t)>1 / n$, we set $s=\alpha^{\sharp}$ and $r=\alpha$ to obtain $0<\lambda=$ $(1 / p) /\left(1 / \alpha-1 / \alpha^{\sharp}\right)<1$. while, if $(1 / \alpha)(1+1 / t) \leqq 1 / n$, we take such $s$ that $1 / \alpha-1 / p>1 / s$ to obtain $0<\lambda=(1 / p) /(1 / \alpha-1 / s)<1$. With these choices of $s$ and $r$ Lemma 2.2 implies

$$
\|v\|_{s}^{\alpha} \leqq c_{0} \| m^{-1} \psi_{t} \int_{\Omega} m|\nabla v|^{\alpha} d x
$$


Putting $\varepsilon^{\prime}=\varepsilon\left\|m^{-1}\right\|_{t}$ we obtain the desired conclusion.

Q.E.D.

Lemma 2.5 ([10]). Let $A$ be a bounded open convex set in $R^{n}$ and let $v \in H^{1, p}(A)(1<p<n)$. Then

$$
\left\{\int_{A}\left|v-v_{A}\right|^{p^{*}} d x\right\}^{1 / p^{*}} \leqq K \frac{(\operatorname{diam} A)^{n}}{\operatorname{meas} A}\left\{\int_{A}|\nabla v|^{p} d x\right\}^{1 / p}
$$

where $v_{A}=\frac{1}{\text { meas } A} \int_{A} v d x$ and where $K$ is a constant depending only on $n$ and $p$.

LEMMA 2.6. Let $v \in H^{1, \alpha}(m, \Omega), \alpha / n>1 / t+\alpha / p$ and assume that

$$
\frac{1}{\operatorname{meas} I(\rho)} \int_{I(\rho)} v d x=0
$$

for an open ball $I(\rho) \subset \Omega$. Then

$$
\left(\rho^{-n} \int_{I(\rho)}|v|^{\alpha \rho /(p-\alpha)} d x\right)^{(p-\alpha) / p} \leqq C\left(\rho^{-n / t}\left\|m^{-1}\right\|_{t}\right) \rho^{\alpha-n} \int_{I(\rho)} m|\nabla v|^{\alpha} d x,
$$

where $C$ is a constant depending on $n, p, t, \alpha$ and $\alpha^{\ddagger}$.

Proof. If $(1 / \alpha)(1+1 / t)>1 / n$, Lemma 2.5 and Hölder's inequality imply

$$
\begin{aligned}
\left(\rho^{-n} \int_{I(\rho)}|v|^{\alpha p /(p-\alpha)} d x\right)^{(p-\alpha) / p} & \leqq \rho^{-n(p-\alpha) / p}\left(\int_{I(\rho)}|v|^{\alpha \#} d x\right)^{\alpha / \alpha \#}[\operatorname{meas} I(\rho)]^{(p-\alpha) / p-\alpha / \alpha^{\sharp}} \\
& \leqq C \rho^{-\alpha n / \alpha^{\sharp}} \rho^{n / t}\left(\rho^{-n / t}\left\|m^{-1}\right\|_{t}\right) \int_{I(\rho)} m|\nabla v|^{\alpha} d x,
\end{aligned}
$$

where $-\left(\alpha n / \alpha^{\sharp}\right)+n / t=\alpha-n$.

If $(1 / \alpha)(1+1 / t) \leqq n / 1$, we choose such $\beta$ that $(p-\alpha) /(\alpha p)>(1 / \beta)(1+1 / t)$ $-1 / n>0$. Then, $\alpha>\beta$ and $\alpha^{\sharp}>\alpha p /(p-\alpha)$. Thus, we see

$$
\begin{aligned}
\left(\int_{I(\rho)}|v|^{\beta \sharp} d x\right)^{\alpha / \beta \#} & \leqq C\left(\int_{I(\rho)}|\nabla v|^{\beta t /(t+1)} d x\right)^{\alpha(t+1) / \beta t} \\
& \leqq C\left(\int_{I(\rho)}|\nabla v|^{\alpha t /(t+1)} d x\right)^{(t+1) / t}[\operatorname{meas} I(\rho)]^{(1-\beta / \alpha) \alpha(t+1) / \beta t} \\
& \leqq C \rho^{n(1-\beta / \alpha) \alpha(t+1) / \beta t}\left\|m^{-1}\right\|_{t} \int_{I(\rho)} m|\nabla v|^{\alpha} d x
\end{aligned}
$$

Therefore we have by Hölder's inequality

$$
\begin{aligned}
\left(\rho^{-n} \int_{I(\rho)}|v|^{\alpha p /(p-\alpha)} d x\right)^{p /(p-\alpha)} & \leqq C \rho^{-\alpha / \beta^{\sharp}}\left(\int_{I(\rho)}|v|^{\beta^{\sharp}} d x\right)^{\alpha / \beta^{\sharp}} \\
& \leqq C \rho^{\alpha-n}\left(\rho^{-n / t}\left\|m^{-1}\right\|_{t}\right) \int_{I(\rho)} m|\nabla v|^{\alpha} d x \text {. Q.E.D. }
\end{aligned}
$$


LEMma 2.7. ([5]). Let $G(x)$ be a uniformly Lipshitz function on $R^{1}$ such that $G(0)=0$. If $v(x) \in H_{0}^{1, \alpha}(m, \Omega)$, then $G(v(x))$ again belongs to $H_{0}^{1, \alpha}(m, \Omega)$. Further, if the derivative $G^{\prime}$ of $G$ is continuous except a finite number of points in $R^{1}$, then we have $G(v)_{x_{i}}=G^{\prime}(v) v_{x_{i}}$ in the sense of distribution.

\section{§3. Global estimates}

In this section we shall prove the maximum principle for weak solutions of the system (1.1).

Definition. Let

$$
H^{1, \alpha}(\tilde{a}, \Omega)=\prod_{j=1}^{m} H^{1, \alpha}\left(\tilde{a}_{j}, \Omega\right) \quad \text { and } \quad H_{0}^{1, \alpha}(\tilde{a}, \Omega)=\prod_{j=1}^{m} H_{0}^{1, \alpha}\left(\tilde{a}_{j}, \Omega\right) .
$$

We say that $u=\left(u_{1}, \cdots, u_{m}\right)$ is a weak solution of the system (1.1), if $u \in H^{1, \alpha}(\tilde{a}, \Omega)$ and if the equation

$$
\sum_{j=1}^{m} \int_{\Omega}\left\{\nabla \Phi_{j} \cdot A_{j}\left(x, u, \nabla u_{j}\right)+\Phi_{j} B_{j}\left(x, u, \nabla u_{j}\right)\right\} d x=0
$$

holds for any $\Phi=\left(\Phi_{1}, \cdots, \Phi_{m}\right) \in C_{0}^{\infty}(\Omega) \times \cdots \times C_{0}^{\infty}(\Omega)$.

By Lemma 2.4, if $u \in H^{1, \alpha}(\tilde{a}, \Omega)$, then $u$ is locally in $L^{\alpha p /(p-1)}(\Omega) \times \cdots$ $\times L^{p /(p-\alpha)}(\Omega)$ for any $p>0$ with $\alpha / n>\alpha / p+1 / t$ and $2 \leqq \alpha<p$. Thus, from the assumption on the coefficients of the structure (1.2), we see that for any $\Phi \in H^{1, \alpha}(\tilde{a}, \Omega)$ with compact support in $\Omega$

$$
\sum_{j=1}^{m} \int_{\Omega}\left\{\left|\Phi_{j} A_{j}\left(x, u, \nabla u_{j}\right)\right|+\left|\Phi_{\jmath} B_{j}\left(x, u, \nabla u_{j}\right)\right|\right\} d x<\infty .
$$

Therefore it follows that if $u$ is a weak solution of (1.1), then (3.1) holds not only for $\Phi \in C_{0}^{\infty}(\Omega) \times \cdots \times C_{0}^{\infty}(\Omega)$, but in fact for any $\Phi \in H^{1, \alpha}(\tilde{a}, \Omega)$ with compact support in $\Omega$.

For a function $u=\left(u_{1}, \cdots, u_{m}\right)$ belonging to $H^{1, \alpha}(\tilde{a}, \Omega)$, we shall simply say $u=0$ on the boundary $\partial \Omega$ of $\Omega$ if $u_{j}, j=1,2, \cdots, m$, belong to the space $H_{0}^{1, \alpha}\left(\tilde{a}_{3}, \Omega\right)$. Similarly, $u=M$ for $M=\left(M_{1}, \cdots, M_{m}\right) \in R^{m}$ on a boundary $\partial \Omega$ of $\Omega$, if $u_{j}-M_{j} \in H_{0}^{1, \alpha}\left(\tilde{a}_{j}, \Omega\right), j=1,2, \cdots, m$.

TheORem 3.1. Let $u=\left(u_{1}, \cdots, u_{m}\right)$ be a weak solution of (1.1) such that $u=0$ on $\partial \Omega$. Then there exists a constant $C$ depending only on $n, p$, $t, \alpha$ and the coefficients of the structure (1.2) such that

$$
\sup _{\Omega}\left|u_{j}\right| \leqq \sum_{i=1}^{m} \sup _{\Omega}\left|u_{i}\right| \leqq C \sum_{i=1}^{m}\left(\left\|u_{i}\right\|_{\alpha}+\left\|f_{i}\right\|_{p / \alpha}^{1 / \alpha}+\left\|g_{i}\right\|_{p / \alpha}^{1 /(\alpha-1)}\right) .
$$


Proof. Without loss of generality, we may assume that meas $\Omega=1$. For, if meas $\Omega \neq 1$, then we can introduce new variable $x^{\prime}=x$ (meas $\left.\Omega\right)^{1 / n}$, so that $u$ satisfies the system of the form (1.1) in a domain $\Omega^{\prime}$ with meas $\Omega^{\prime}=1$.

Now we put $\kappa_{j}=\left\|f_{j}\right\|_{p / \alpha}^{1 / \alpha}+\left\|g_{j}\right\|_{p / \alpha}^{1 /(\alpha-1)}+\varepsilon$ for any positive number $\varepsilon$ and $\bar{u}_{j}=\left|u_{j}\right|+\kappa_{j}$, and define the functions

$$
G_{j}\left(u_{j}\right)= \begin{cases}\bar{u}_{j}^{\alpha q-\alpha+1}-\kappa_{j}^{\alpha q-\alpha+1} & \text { for }\left|u_{j}\right| \leqq \ell_{j}-\kappa_{j}, \\ \ell_{j}^{\alpha q-\alpha} \bar{u}_{j}-\kappa_{j}^{\alpha q-\alpha+1} & \text { for }\left|u_{j}\right| \geqq \ell_{j}-\kappa_{j},\end{cases}
$$

$j=1,2, \cdots, m$, where $q \geqq 1$ is any fixed number and $\ell_{j}$ are constants greater than $\kappa_{j}$.

Next, we define $\Phi_{j}=G_{j} \operatorname{sign}\left(u_{j}\right), j=1, \cdots, m$. It is clear that $\Phi=$ $\left(\Phi_{1}, \cdots, \Phi_{m}\right) \in H_{0}^{1, \alpha}(\tilde{a}, \Omega)$. Thus, we have

$$
\sum_{j=1}^{m} \int_{\Omega}\left\{\nabla \Phi_{j} \cdot A_{j}+\Phi_{j} B_{j}\right\} d x=0
$$

If we put

$$
H_{j}\left(u_{j}\right)= \begin{cases}(\alpha q-\alpha+1) \bar{u}_{j}^{\alpha q-\alpha} & \text { for }\left|u_{\jmath}\right| \leqq \ell_{j}-\kappa_{j}, \\ \ell_{j}^{\alpha q-\alpha} & \text { for }\left|u_{j}\right| \geqq \ell_{j}-\kappa_{j},\end{cases}
$$

then we see

$$
\nabla \Phi_{j}=H_{j} \nabla u_{j}, \quad\left|\nabla u_{j}\right|=\left|\nabla \bar{u}_{j}\right| \text { and } \quad G_{\jmath} \leqq \bar{u}_{j}^{q} H_{j}^{(\alpha-1) / \alpha} .
$$

Therefore, by (1.2), we have

$$
\begin{aligned}
\sum_{l=1}^{m} \int_{\Omega} H_{j} a_{j}\left|\nabla u_{j}\right|^{\alpha} d x \leqq \sum_{j=1}^{m} \int_{\Omega} & \left\{G_{j} b_{\jmath}\left|\nabla \bar{u}_{j}\right|^{\alpha-1}+H_{j} \sum_{i=1}^{m} c_{i j} \bar{u}_{\imath}^{\alpha}\right. \\
& \left.+G_{j} \sum_{i=1}^{m} d_{i j} \bar{u}_{i}^{\alpha-1}+H_{j} f_{j}+G_{j} g_{\jmath}\right\} d x
\end{aligned}
$$

and

$$
G_{j} b_{j}\left|\nabla \bar{u}_{j}\right|^{a-1} \leqq \frac{1}{2} H_{j} a_{j}\left|\nabla \bar{u}_{j}\right|^{\alpha}+2^{\alpha-1} b_{j}^{\alpha} a_{j}^{1-\alpha} \bar{u}_{j}^{\alpha q}
$$

Moreover, since $G_{j} \leqq \bar{u}_{j}^{\alpha q-\alpha+1}, \quad H_{j} \leqq(\alpha q-\alpha+1) \bar{u}_{j}^{\alpha q-\alpha}, \quad \bar{u}_{j}^{\alpha q-\alpha+1} \bar{u}_{\imath}^{\alpha-1} \leqq$ $\bar{u}_{j}^{\alpha q}+\bar{u}_{i}^{\alpha q}$ and $\bar{u}_{j}^{\alpha q-\alpha} \bar{u}_{i}^{\alpha} \leqq \bar{u}_{j}^{\alpha q}+\bar{u}_{i}^{\alpha q}$, we have

$$
H_{j} \sum_{i=1}^{m} c_{i j} \bar{u}_{i}^{\alpha}+G_{j} \sum_{i=1}^{m} d_{i j} \bar{u}_{i}^{\alpha-1} \leqq(\alpha q-\alpha+1) \sum_{i=1}^{m}\left(c_{i j}+d_{i j}\right)\left(\bar{u}_{i}^{\alpha q}+\bar{u}_{j}^{\alpha q}\right) .
$$

We also have 


$$
H_{j} f_{j}+G_{j} g_{j} \leqq(\alpha q-\alpha+1)\left(\frac{f_{j}}{\kappa_{j}^{\alpha}}+\frac{g_{j}}{\kappa_{j}^{\alpha-1}}\right) \bar{u}_{j}^{\alpha q}
$$

since $\kappa_{j} \leqq \bar{u}_{j}$.

Therefore, it follows from (3.3) that

$$
\sum_{j=1}^{m} \int_{\Omega} H_{j} a_{j}\left|\nabla \bar{u}_{j}\right|^{\alpha} d x \leqq C(\alpha q-\alpha+1) \sum_{j=1}^{m} \int_{\Omega} B_{j}(x) \bar{u}_{\jmath}^{\alpha q} d x
$$

where

$$
B_{j}(x)=b_{j}^{\alpha} a_{\jmath}^{1-\alpha}+\sum_{i=1}^{m}\left(c_{i j}+c_{j i}+d_{i j}+d_{j i}\right)+\frac{f_{j}}{\kappa_{j}^{\alpha}}+\frac{g_{j}}{\kappa_{\jmath}^{\alpha-1}} \in L^{\alpha / p}(\Omega)
$$

and $C$ is a constant depending only on $\alpha$.

The right-hand side of (3.4) is independent of $\ell_{j} \geqq 0(j=1, \cdots, m)$. Since $H_{j}$ are non-decreasing functions and since $\lim _{\ell_{j} \rightarrow \infty} H_{j}=(\alpha q-\alpha+1) \bar{u}_{j}^{\alpha q}$, the monotone convergence theorem proves that

$$
\sum_{j=1}^{m} \int_{\Omega} a_{j}\left(\bar{u}_{j}^{q-1}\left|\nabla \bar{u}_{j}\right|\right)^{\alpha} d x \leqq C \sum_{j=1}^{m} \int_{\Omega} B_{j}(x) \bar{u}_{j}^{\alpha q} d x
$$

Since $\left|\nabla\left(\bar{u}_{j}^{q}\right)\right|=q \bar{u}_{j}^{q-1}\left|\nabla \bar{u}_{j}\right|$, putting $v_{j}=\bar{u}_{j}^{q}$, we have

$$
\sum_{j=1}^{m} \int_{\Omega} a_{j}\left|\nabla v_{j}\right|^{\alpha} d x \leqq C q^{\alpha} \sum_{j=1}^{m} \int_{\Omega} B_{j}(x) v_{j}^{\alpha} d x
$$

By Hölder's inequality we have

$$
\int_{\Omega} B_{j}(x) v_{j}^{\alpha} d x \leqq\left\|B_{j}\right\|_{p / \alpha}\left\|v_{j}\right\|_{\alpha p /(p-\alpha)}^{\alpha} \leqq\left\|B_{j}\right\|_{p / \alpha}\left(\left\|v_{j}-\kappa_{j}^{q}\right\|_{\alpha p /(p-\alpha)}+\kappa_{j}^{\alpha q}\right) .
$$

Since $v_{j}-\kappa_{j}^{q} \in H_{0}^{1, \alpha}\left(a_{j}, \Omega\right)$ and $v_{i}-\kappa_{j}^{\alpha} \leqq v_{j}$, by Lemma 2.4, we have

$$
\left\|v_{j}-\kappa_{j}^{q}\right\|_{\alpha p /(p-\alpha)}^{\alpha} \leqq 2^{-1} C^{-1} q^{-\alpha}\left\|B_{i}\right\|_{p / \alpha}^{-1} \int_{\Omega} a_{j}\left|\nabla v_{j}\right|^{\alpha} d x+C q^{\alpha \gamma}\left\|v_{j}\right\|_{\alpha}^{\alpha},
$$

and since $\kappa_{\jmath}^{q} \leqq v_{\jmath}$ and meas $\Omega=1$, it follows that $\kappa_{j}^{\alpha q} \leqq\left\|v_{j}\right\|_{\alpha}^{\alpha}$. Thus we have

$$
\sum_{j=1}^{m} \int_{\Omega} a_{j}\left|\nabla v_{j}\right|^{\alpha} d x \leqq C q^{\alpha \gamma} \sum_{j=1}^{m}\left\|v_{j}\right\|_{\alpha}^{\alpha},
$$

where $C$ is a constant depending only on $n, p, t, \alpha$ and $\| B_{j} p / \alpha,(j=1$, $\cdots, m)$.

By Lemma 2.2 and (3.5) we see the inequalities 


$$
\begin{aligned}
\left\|\sum_{j=1}^{m} v_{j}\right\|_{\alpha \#}^{\alpha} & \leqq C \sum_{j=1}^{m}\left(\left\|v_{j}-\kappa_{j}^{q}\right\|_{\alpha \sharp}^{\alpha}+\left\|v_{j}\right\|_{\alpha}^{\alpha}\right) \\
& \leqq C \sum_{j=1}^{m}\left(\int_{\Omega} a_{j}\left|\nabla v_{j}\right|^{\alpha} d x+\left\|v_{j}\right\|_{\alpha}^{\alpha}\right) \\
& \leqq C q^{\alpha \gamma}\left\|\sum_{j=1}^{m} v_{j}\right\|_{\alpha}^{\alpha},
\end{aligned}
$$

which prove

$$
\left(\int_{\Omega}\left(\sum_{j=1}^{m} \bar{u}_{j}\right)^{\alpha q \alpha^{\sharp} / \alpha} d x\right)^{1 / \alpha \# q} \leqq C^{1 / \alpha q} q^{\gamma(1 / q)}\left(\int_{\Omega}\left(\sum_{j=1}^{m} \bar{u}_{j}\right)^{\alpha q} d x\right)^{1 / \alpha q} .
$$

Thus, putting $r=\alpha^{\sharp} / \alpha$ and $q=r^{s}, s=0,1,2, \cdots$, we have

$$
\begin{aligned}
\left\|\sum_{j=1}^{m} \bar{u}_{j}\right\|_{\alpha r^{s+1}} & \leqq C^{(1 / \alpha) r^{s}} r^{\gamma s / r s}\left\|\sum_{j=1}^{m} \bar{u}_{j}\right\|_{\alpha r s} \\
& \leqq C^{(1 / \alpha)\left(\sum_{s=0}^{\infty} 1 / r^{s}\right)} r^{r\left(\sum_{s=0}^{\infty} s / r^{s}\right)}\left\|\sum_{j=1}^{m} \bar{u}_{j}\right\|_{\alpha} \\
& \leqq C \sum_{j=1}^{m}\left\|\bar{u}_{j}\right\|_{\alpha} .
\end{aligned}
$$

Now, let $s$ tend to infinity to have

$$
\sup _{\Omega} \sum_{j=1}^{m} \bar{u}_{j} \leqq C \sum_{j=1}^{m}\left\|\bar{u}_{j}\right\|_{\alpha},
$$

Note that $\left\|f_{j} / \kappa_{j}^{\alpha}+g_{j} / \kappa_{j}^{\alpha-1}\right\|_{p / \alpha} \leqq 1$ for any $\varepsilon>0$. Therefore letting $\varepsilon$ tend to zero, we have (3.2).

TheOREM 3.2. Let $u=\left(u_{1}, \cdots, u_{m}\right)$ be a weak solution of (1.1) such that $u=M$ on $\partial \Omega$ for $M=\left(M_{1}, \cdots, M_{m}\right) \in R^{m}$. Then it holds

$$
\sup _{\Omega}\left|u_{j}\right| \leqq C \sum_{i=1}^{m}\left(\left\|u_{i}\right\|_{\alpha}+\left\|f_{i}\right\|_{p / \alpha}^{1 / \alpha}+\left\|g_{i}\right\|_{p / \alpha}^{1 /(\alpha-1)}+\left|M_{i}\right|\right)
$$

where $C$ is a constant depending only on $n, p t, \alpha$ and the coefficients of the structure (1.2).

Proof. Consider $U=u-M=\left(u_{1}-M_{1}, \cdots, u_{m}-M_{m}\right)$. Then $U=0$ on $\partial \Omega$. Since $u$ is a weak solution of (1.1), we must have

$$
-\operatorname{div} \tilde{A}_{j}\left(x, U, \nabla U_{j}\right)+\tilde{B}_{j}\left(x, U, \nabla U_{j}\right)=0 \quad j=1,2, \cdots, m,
$$

where

$$
\tilde{A}_{j}\left(x, U, \nabla U_{j}\right)=A_{j}\left(x, U+M, \nabla U_{j}\right) \text { and } \tilde{B}_{j}\left(x, U, \nabla U_{j}\right)=B_{j}\left(x, U+M, \nabla U_{j}\right) .
$$

In view of (1.2) 


$$
\left\{\begin{array}{l}
\xi \cdot \tilde{A}_{j}(x, U, \xi) \geqq a_{j}|\xi|^{\alpha}-\sum_{i=1}^{m} c_{\imath \jmath}\left|U_{j}+M_{j}\right|^{\alpha}-f_{j}, \\
\left|B_{\jmath}(x, U, \xi)\right| \leqq b_{j}|\xi|^{\alpha-1}+\sum_{i=1}^{m} d_{i \jmath}\left|U_{\imath}+M_{i}\right|^{\alpha-1}+g_{j},
\end{array}\right.
$$

$j=1,2, \cdots, m$, for any $\xi \in R^{n}$. From these, we immediately have the following;

There exist positive constants $\lambda$ and $\mu$ depending only on $\alpha$ such that for any $\xi \in R^{n}$

$$
\left\{\begin{array}{l}
\xi \cdot \tilde{A}_{j}(x, U, \xi) \geqq a_{\jmath}|\xi|^{\alpha}-\sum_{i=1}^{m} \lambda c_{i j}\left|U_{\imath}\right|^{\alpha}-F_{\jmath} \\
\left|\tilde{B}_{j}(x, U, \xi)\right| \leqq b,|\xi|^{\alpha-1}+\sum_{i=1}^{m} \mu d_{i j}\left|U_{i}\right|^{\alpha-1}+G_{\jmath}
\end{array}\right.
$$

where

$$
F_{\jmath}=f_{\jmath}+\sum_{i=1}^{m} \lambda M_{\imath}^{\alpha} c_{i \jmath}, \quad G_{j}=g_{j}+\sum_{i=1}^{m} \mu M_{i}^{\alpha-1} d_{\imath j} \in L^{p / \alpha}(\Omega) .
$$

Therefore we can apply (3.6) to obtain

$$
\sup _{\Omega} \bar{U}_{J} \leqq \sup _{\Omega}\left(\sum_{i=1}^{m} \bar{U}_{j}\right) \leqq C \sum_{i=1}^{m}\left\|\bar{U}_{j}\right\|_{\alpha}
$$

for $\bar{U}_{j}=\left|U_{j}\right|+\left\|F_{j}\right\|_{p / \alpha}^{1 / \alpha}+\left\|G_{j}\right\|_{p / \alpha}^{1 / \alpha-1)}+\varepsilon$. Since constant $C$ does not depend on $\left\|F_{,}\right\|_{p / \alpha}^{1 / \alpha}+\left\|G_{j}\right\|_{p / \alpha}^{1 /(\alpha-1)}+\varepsilon$, we obtain (3.7), by letting $\varepsilon$ tend to zero.

Q.E.D.

In the particular case where $m=1$, more sharp results can be obtained.

THEOREM 3.3. Let $u$ be a weak solution of (1.1) where $m=1$ and let $u \leqq M$ on $\partial \Omega$. Then it holds that

$$
\sup _{\Omega} u \leqq(1+C) M^{+}+C\left\{\|u\|_{\alpha}+\|f\|_{p / \alpha}^{1 / \alpha}+\|g\|_{p / \alpha}^{1 /(\alpha-1)}\right\},
$$

where $M^{+}=\max (0, M)$ and $C$ is a constant depending only on $n, p, t, \alpha$ and the coefficients of the structure (1.2).

\section{$\S 4$. Local boundedness}

In this section, we shall derive the local estimates both in the interior and near the boundary of $\Omega$ for the weak solutions of (1.1).

Let $I\left(x_{0}, \rho\right)=I(\rho)$ be an open ball with center at $x_{0}$, radius $\rho$ and let $\Omega\left(x_{0}, \rho\right)=\Omega \cap I\left(x_{0}, \rho\right)$. We set 


$$
\begin{aligned}
B_{j}= & B_{j}(x)=\tilde{a}_{j}+\tilde{a}_{j}^{\alpha} a_{j}^{1-\alpha}+b_{j}^{\alpha} a_{j}^{1-\alpha}+\sum_{i=1}^{m}\left\{c_{i j}+c_{j i}+d_{i j}+d_{j i}\right. \\
& \left.+\left(e_{\imath j}^{\alpha /(\alpha-1)}+e_{j i}^{\alpha /(\alpha-1)}\right) \tilde{a}_{j}^{1 /(1-\alpha)}\right\}+\kappa_{j}^{-\alpha}\left(f_{j}+h_{j}^{\alpha /(1-\alpha)} \tilde{a}_{j}^{1 /(1-\alpha)}\right)+\kappa_{j}^{1-\alpha} g_{j}
\end{aligned}
$$

for positive constants $\kappa_{j}$. Moreover we put $\tilde{p}=(p / \alpha)^{\prime}=p /(p-\alpha)$,

$$
a_{j}(\rho)=\left(\rho^{-n} \int_{I(p)} a_{j}(x)^{-t} d x\right)^{1 / t}, \quad B_{j}(\rho)=\left(\rho^{-n} \int_{I(\rho)} B_{j}(x)^{p / \alpha} d x\right)^{\alpha / p},
$$

and

$$
\zeta=\zeta\left(x, \rho^{\prime}, \rho\right)=\left\{\begin{array}{cl}
1 & \text { in } I\left(\rho^{\prime}\right) \\
\frac{\rho-|x|}{\rho-\rho^{\prime}} & \text { for } \rho^{\prime} \leqq|x| \leqq \rho \\
0 & \text { outside of } I(\rho)
\end{array}\right.
$$

for $0<\rho^{\prime}<\rho$.

We obtain the following lemma by the same argument as in the proof of Lemma 2.2, using Hölder's inequality

Lemma 4.1. Let $v \in H^{1, \alpha}\left(a_{j}, \Omega\right)$. Then for any $\alpha$ with $1+1 / t<\alpha<p$, and for any $\rho_{0}$ with $0<\rho^{\prime}<\rho \leqq \rho_{0}$

$$
\begin{aligned}
\left(\rho_{0}^{-n} \int_{I\left(\rho^{\prime}\right)}|v|^{\left.\right|^{\sharp}} d x\right)^{\alpha / \alpha^{\sharp}} \leqq & c_{0} \rho_{0}^{\alpha-n} a_{j}\left(\rho_{0}\right) \int_{I(p)} a_{\jmath}|\nabla v|^{\alpha} \zeta^{\alpha} d x \\
& +\rho_{0}^{\alpha}\left(\rho-\rho^{\prime}\right)^{-\alpha}\left(\rho_{0}^{-n} \int_{I(\rho)}|v|^{\alpha \tilde{p}} d x\right)^{1 / \tilde{p}}
\end{aligned}
$$

where $\zeta=\zeta\left(x, \rho^{\prime}, \rho\right)$.

ThEOREM 4.1. Let $u=\left(u_{1}, \cdots, u_{m}\right)$ be a weak solution of (1.1), and $I\left(x_{0}, 2 \rho_{0}\right) \subset \Omega$. Then it holds that

$$
\sup _{I\left(x_{0}, \rho_{0}\right)}\left|u_{j}\right| \leqq C \sum_{i=1}^{m}\left\{\left(\rho_{0}^{-n} \int_{I\left(x_{0}, 2 \rho 0\right)}\left|u_{i}\right|^{\alpha \tilde{p}} d x\right)^{1 / \alpha \tilde{p}}+\rho_{0}^{-n / \alpha \tilde{p}} K_{i}\right\}
$$

where

$$
\begin{array}{r}
K_{i}=\left(\int_{I(2 \rho 0)} f_{i}^{p / \alpha} d x\right)^{1 / p}+\left(\int_{I(2 \rho 0)} g_{i}^{p / \alpha} d x\right)^{\alpha /(\alpha-1) p} \\
\quad+\left(\int_{I(2 \rho 0)}\left(h_{i}^{\alpha /(\alpha-1)} \tilde{a}_{i}^{1 /(1-\alpha)}\right)^{p / \alpha} d x\right)^{\alpha /(\alpha-1) p}
\end{array}
$$

and $C$ is a constant depending only on $n, p, t, \alpha, a_{j}\left(2 \rho_{0}\right)$ and $B_{j}\left(2 \rho_{0}\right)(j=1$, $\cdots, m)$.

Proof. Put $\kappa_{\jmath}=K_{\jmath}+\varepsilon$ for a positive number $\varepsilon$ and put $\bar{u}_{i}=\left|u_{j}\right|+\kappa_{j}$ 
$(j=1, \cdots, m)$. Define $\Phi_{j}$ by $\Phi_{j}=\operatorname{sign}\left(u_{j}\right) G_{j} \zeta^{\alpha}\left(x, \rho^{\prime}, \rho\right)$ for $0<\rho^{\prime}<\rho \leqq 2 \rho_{0}$, where

$$
G_{\jmath}=G_{j}\left(u_{j}\right)= \begin{cases}\bar{u}_{j}^{\alpha q-\alpha+1}-\kappa_{j}^{\alpha q-\alpha+1} & \text { for }\left|u_{j}\right| \leqq \ell_{j}-\kappa_{j}, \\ \ell_{j}^{\alpha q-\alpha} u_{j}-\kappa_{j}^{\alpha q-\alpha+1} & \text { for }\left|u_{j}\right| \geqq \ell_{j}-\kappa_{j},\end{cases}
$$

$j=1,2, \cdots, m, q \geqq 1, \ell_{j}>\kappa_{j}$.

Then we have

$$
\sum_{j=1}^{m} \int_{I(\rho)}\left\{\nabla \Phi_{j} \cdot A_{j}\left(x, u, \nabla u_{j}\right)+\Phi_{j} B_{j}\left(x, u, \nabla u_{j}\right)\right\} d x=0 .
$$

Put

$$
H_{j}= \begin{cases}(\alpha q-\alpha+1) \bar{u}_{j}^{\alpha q-\alpha} & \text { for }\left|u_{j}\right| \leqq \ell_{j}-\kappa_{j}, \\ \ell_{j}^{\alpha q-\alpha} & \text { for }\left|u_{j}\right| \geqq \ell_{j}-\kappa_{j} .\end{cases}
$$

Since $\nabla \Phi_{j}=H_{j} \zeta^{\alpha} \nabla u_{j}+\alpha G_{j} \zeta^{\alpha-1} \nabla \zeta$, we have, by (1.2),

$$
\begin{aligned}
\sum_{j=1}^{m} \int_{I(\rho)} & H_{j} a_{j}\left|\nabla \bar{u}_{j}\right|^{\alpha} \zeta^{\alpha} d x \\
\leqq & \sum_{j=1}^{m} \int_{I(\rho)}\left\{\alpha G_{j} \zeta^{\alpha-1}|\nabla \zeta|\left(\tilde{a}_{j}\left|\nabla \bar{u}_{j}\right|^{\alpha-1}+\sum_{i=1}^{m} e_{i j} \bar{u}_{i}^{\alpha-1}+h_{j}\right)\right. \\
& \left.\quad+G_{j} \zeta^{\alpha}\left(b_{j}\left|\nabla \bar{u}_{j}\right|^{\alpha-1}+\sum_{i=1}^{m} d_{i j} \bar{u}_{i}^{\alpha-1}+g_{j}\right)+H_{j} \zeta^{\alpha} \sum_{i=1}^{m} c_{i j} \bar{u}_{i}^{\alpha}+H_{j} \zeta^{\alpha} f_{j}\right\} d x .
\end{aligned}
$$

It follows by Young's inequality that

$$
\begin{aligned}
& G_{\jmath} \zeta^{\alpha-1}|\nabla \zeta| \tilde{a}_{j}\left|\nabla \bar{u}_{j}\right|^{\alpha-1} \leqq \frac{H_{j}}{4} a_{j}\left|\nabla \bar{u}_{j}\right|^{\alpha} \zeta^{\alpha}+4^{\alpha-1} \tilde{a}_{\jmath}^{\alpha} a_{\jmath}^{1-\alpha} \bar{u}_{j}^{\alpha q}|\nabla \zeta|^{\alpha}, \\
& G_{j} \zeta^{\alpha-1}|\nabla \zeta| e_{i j} \bar{u}_{i}^{\alpha-1} \leqq e_{i j}^{\alpha /(\alpha-1)} \tilde{a}_{\jmath}^{1 /(1-\alpha)} \bar{u}_{i}^{\alpha q} \zeta^{\alpha}+\tilde{a}_{j} \bar{u}_{\jmath}^{\alpha q}|\nabla \zeta|^{\alpha}
\end{aligned}
$$

and

$$
\begin{aligned}
G_{j} \zeta^{\alpha-1}|\nabla \zeta| h_{j} & \leqq G_{j} \zeta^{\alpha-1}|\nabla \zeta| h_{j} \kappa_{j}^{1-\alpha} \bar{u}_{j}^{\alpha-1} \\
& \leqq \kappa_{j}^{-\alpha} h^{\alpha /(\alpha-1)} \tilde{a}_{j}^{1 /(1-\alpha)} \bar{u}_{j}^{\alpha q} \zeta^{\alpha}+\tilde{a}_{j} \bar{u}_{j}^{\alpha q}|\nabla \zeta|^{\alpha} .
\end{aligned}
$$

Put $v_{j}=\bar{u}_{j}^{q}$. By the same argument as in Theorem 3.1, we see that

$$
\begin{aligned}
\sum_{j=1}^{m} \int_{I(\rho)} a_{j}\left|\nabla v_{j}\right|^{\alpha} \zeta^{\alpha} d x & \leqq C q^{\alpha}\left(\rho-\rho^{\prime}\right)^{-\alpha} \sum_{j=1}^{m} \int_{I(\rho)} B_{j}(x) v_{j}^{\alpha} d x \\
& \leqq C q^{\alpha}\left(\rho-\rho^{\prime}\right)^{-\alpha} \rho_{0}^{\alpha} \sum_{j=1}^{m} B_{j}\left(2 \rho_{0}\right)\left(\rho_{0}^{-n} \int_{I(\rho)} v_{j}^{\alpha \tilde{p}} d x\right)^{1 / \tilde{p}} .
\end{aligned}
$$

This result, Lemma 4.1 and (4.2) imply

$$
\left(\rho_{0}^{-n} \int_{I\left(\rho^{\prime}\right)} v_{j}^{\alpha \sharp} d x\right)^{\alpha / \alpha \#} \leqq C q^{\alpha}\left(\rho-\rho^{\prime}\right)^{-\alpha} \rho_{0}^{\alpha}\left(\rho_{0}^{-n} \int_{I(\rho)} v_{j}^{\alpha \tilde{p}} d x\right)^{1 / \tilde{p}},
$$


where $C$ is a constant depending only on $n, p, t, \alpha, \alpha^{\sharp}, a_{j}\left(2 \rho_{0}\right)$ and $\sum_{j=1}^{m} B_{j}\left(2 \rho_{0}\right)$.

Now put $r=\alpha / \alpha^{\ddagger}, q_{s}=(r / p)^{s}$ and $\rho_{s}=\rho_{0}+2^{-s} \rho_{0}(s=0,1,2, \cdots)$.

Then

$$
\begin{aligned}
\left(\rho_{0}^{-n}\right. & \left.\int_{I\left(\rho_{s+1}\right)}\left(\sum_{j=1}^{m} \bar{u}_{j}\right)^{\alpha q_{s+1} \tilde{p}} d x\right)^{1 / \alpha q_{s+1} \tilde{p}} \\
& =\left(\rho_{0}^{-n} \int_{I\left(\rho_{s+1}\right)}\left(\sum_{j=1}^{m} \bar{u}_{j}\right)^{\alpha / q_{s} r} d x\right)^{1 / \alpha q_{s+1} \tilde{p}} \\
& \leqq C^{1 / q_{s+1} \tilde{p}} 2^{(s+1) / r^{s+1 \tilde{p}}}\left(\rho_{0}^{-n} \int_{I\left(\rho_{s}\right)}\left(\sum_{j=1}^{m} \bar{u}_{j}\right)^{\alpha q_{s} r} d x\right)^{1 / \alpha q_{s} \tilde{p}} \\
& \leqq C^{\left.1 / \alpha \sum_{k=0}^{\infty}(\tilde{p} / r)^{k}\right)} 2^{\left(1 / \alpha \sum_{k=0}^{\infty} k(\tilde{p} / r)^{k)}\right.}\left(\rho_{0}^{-n} \int_{I\left(2 \rho_{0}\right)}\left(\sum_{j=1}^{m} \bar{u}_{j}\right)^{\alpha \tilde{p}} d x\right)^{1 / \alpha \tilde{p}} .
\end{aligned}
$$

from which, letting $s$ tend to infinity and $\varepsilon$ tend to zero, we have the desired inequality (4.1).

Q.E.D.

Theorem 4.2. Let $u=\left(u_{1}, \cdots, u_{m}\right)$ be a weak solution of (1.1) and $x_{0} \in \partial \Omega$. Suppose that for some positive number $\rho_{0}, u=M$ on $\partial \Omega \cap I\left(x_{0}, 2 \rho_{0}\right)$, $M=\left(M_{1}, \cdots, M_{m}\right) \in R^{m}$. Then

$$
\sup _{\Omega\left(x_{0}, \rho_{0}\right)}\left|u_{j}\right| \leqq \rho_{0}^{-\theta} C \sum_{i=1}^{m}\left\{\left(\int_{\Omega\left(x_{0}, 2 \rho_{0}\right)}\left|u_{i}\right|^{\alpha \tilde{p}} d x\right)^{1 / \alpha \tilde{p}}+\left|M_{i}\right|+K_{i}\right\}, \quad j=1, \cdots, m,
$$

where

$$
\begin{aligned}
K_{i}= & \left(\int_{\Omega\left(x_{0}, 2 \rho_{0}\right)} f_{i}^{p / \alpha} d x\right)^{1 / p}+\left(\int_{\Omega\left(x_{0}, 2 \rho_{0}\right)} g_{i}^{p / \alpha} d x\right)^{\alpha /(\alpha-1) p} \\
& +\left(\int_{\Omega\left(x_{0}, 2 \rho_{0}\right)}\left(h_{i}^{\alpha /(\alpha-1)} \tilde{a}_{i}^{1 /(1-\alpha) p / \alpha} d x\right)^{\alpha /(\alpha-1) p} .\right.
\end{aligned}
$$

The constant $C$ depends only on $n, p, t, \alpha,\left\|a_{j}^{-1}\right\|_{t}$ and $\left\|B_{j}\right\|_{p / \alpha}(j=1, \cdots, m)$, while $\theta$ depends on $n, p, t$ and $\alpha$.

Proof. Let $U=u-M=\left(u_{1}-M_{1}, \cdots, u_{m}-M_{m}\right)$. Then $U$ is a weak solution of a system of the form (1.1) such that $U=0$ on $\partial \Omega \cap I\left(x_{0}, 2 \rho_{0}\right)$. Therefore the same method of the proof of Theorem 4.1 can be applied to prove the assertion.

Q.E.D.

\section{§5. Hölder continuity}

In this section we shall prove that weak solutions of (1.1) are Hölder continuous in $\Omega$ under the assumption

$$
\tilde{a}_{j} a_{j}^{-1} \in L^{\infty}(\Omega), \quad j=1,2, \cdots, m .
$$

First we shall state some lemmas. 
Lemma $5.1([9])$. Let $I\left(x_{0}, \rho_{0}\right) \subset \Omega$ and $v$ be an $H^{1,1}\left(I\left(x_{0}, \rho_{0}\right)\right)$-function. Put

$$
A\left(k_{0}, \rho_{0}\right)=\left\{x \in I\left(x_{0}, \rho_{0}\right) \mid v \geqq k_{0}\right\} .
$$

Suppose that there are two constants $k_{0}$ and $\theta(0 \leqq \theta<1)$ such that

$$
\text { meas } A\left(k_{0}, \rho_{0}\right)<\theta \text { meas } I\left(x_{0}, \rho_{0}\right)
$$

holds. Then for any $h$ and $k$ with $h>k>k_{0}$, there exists a positive constant $C$ depending only on $\theta$ and $n$ such that the following inequality hold:

$$
(h-k)\left[\operatorname{meas} A\left(h, \rho_{0}\right)\right]^{(n-1) / n} \leqq C \int_{\left[A\left(k, \rho_{0}\right)-A\left(h, \rho_{0}\right)\right]}|\nabla v(t)| d t .
$$

This lemma immediately implies the following

LEMMA 5.2. Under the same hypothesis of Lemma 5.1, we have

$$
\begin{aligned}
& (h-k)^{\alpha}\left[\operatorname{meas} A\left(h, \rho_{0}\right)\right]^{\alpha(n-1) / n} \leqq c_{0} \rho_{0}^{n / t}\left(\rho_{0}^{-n} \int_{I\left(\rho_{0}\right)} m^{-t} d x\right)^{1 / t} \\
& \times \int_{A\left(k, \rho_{0}\right)} m|\nabla v|^{\alpha} d x\left[\text { meas } A\left(k, \rho_{0}\right)-\operatorname{meas} A\left(h, \rho_{0}\right)\right]^{\alpha-1-(1 / t)}
\end{aligned}
$$

for $v \in H^{1, \alpha}(m, \rho)$.

LEMMA 5.3 ([8]). Let $\phi(h, \rho)$ be a non-negative function on the strip $\left(h \geqq k_{0} \geqq 0\right) \times\left(0 \leqq \rho<R_{0}\right)$ such that

(i) for every fixed $\rho, \phi(h, \rho)$ is non-increasing on $h$

(ii) for every fixed $h,(h, \rho)$ is non-decreasing in $\rho$ and that there exist positive constant $C, \alpha, \beta, \gamma(\beta>1)$ with

$$
\phi(h, \rho) \leqq C[\phi(k, R)]^{\beta}(h-k)^{-\alpha}(R-\rho)^{-\gamma}
$$

for $h>k \geqq k_{0}, \rho<R \leqq R_{0}$. Then we have

$$
\phi\left(k_{0}+d, R_{0}-\sigma R_{0}\right)=0
$$

for any $\sigma$ with $0<\sigma<1$ and for

$$
d=C^{1 / \alpha}\left(\sigma^{-1} R_{0}^{-1}\right)^{\gamma / \alpha}\left[\phi\left(k_{0}, R_{0}\right)\right]^{(\beta-1) / \alpha_{2} \beta(\alpha+\beta) /(\beta-1) \alpha} .
$$

Remark. Lemma 5.3 is valid not only for $k_{0} \geqq 0$, but also $k_{0}<0$.

From now on, we fix $j$ and use simplified notations:

$$
\begin{aligned}
& M(\rho)=M_{j}(\rho)=\sup _{I(\rho)} u_{j}, \quad m(\rho)=m_{j}(\rho)=\inf _{I(\rho)} u_{j}, \\
& \omega(\rho)=\omega_{j}(\rho)=M_{j}(\rho)-m_{j}(\rho) .
\end{aligned}
$$


TheOREm 5.1. Let $u=\left(u_{1}, \cdots, u_{m}\right)$ be a weak solution of (1.1) and $I(R)=I\left(x_{0}, R\right) \subset \Omega$ for $0<4 \rho_{0}<R$. Then under the assumption (5.1) there exist positive constants $K$ and $\lambda$ such that for any $\rho$ with $0<\rho<\rho_{0}$

$$
\omega_{j}(\rho) \leqq K \rho^{\lambda} \quad(j=1,2, \cdots, m),
$$

which means the local Hölder continuity of $u_{j}$ in $\Omega$.

Proof. We put $F_{j}(x)=\sum_{i=1}^{m} c_{i j}\left|u_{i}\right|^{\alpha}+f_{j}, G_{j}(x)=\sum_{i=1}^{m} d_{i j}\left|u_{i}\right|^{\alpha-1}+g_{j}$ and $H_{j}=\sum_{i=1}^{m} e_{i j}\left|u_{i}\right|^{\alpha-1}+h_{j}$. Then from (1.2), we have

$$
\left\{\begin{array}{l}
\xi \cdot A_{j}(x, u, \xi) \geqq a_{j}|\xi|^{\alpha}-F_{j}, \\
\left|B_{j}(x, u, \xi)\right| \leqq b_{j}|\xi|^{\alpha-1}+G_{j}, \\
\left|A_{j}(x, u, \xi)\right| \leqq \tilde{a}_{j}|\xi|^{\alpha-1}+H_{j}
\end{array}\right.
$$

$j=1,2, \cdots, m$, for any $\xi \in R^{n}$. Moreover, since $u=\left(u_{1}, \cdots, u_{m}\right)$ is bounded in $I(R), F_{j}, G_{j}, H_{j}^{\alpha /(\alpha-1)} \tilde{a}_{j}^{1 /(1-\alpha)} \in L^{p / \alpha}(I(R))$.

We define the functions

$$
\left\{\begin{array}{l}
\Phi_{j}=\left(u_{j}-k\right)^{+} \zeta^{\alpha}, \quad \text { for any choice of } k, \\
\Phi_{i}=0 \quad(i \neq j),
\end{array}\right.
$$

where $\zeta=\zeta\left(x, \rho^{\prime}, \rho\right)$ with $\rho_{0}<\rho^{\prime}<\rho \leqq 2 \rho_{0}$ and $\left(u_{j}-k\right)^{+}=\max \left(u_{j}-k, 0\right)$. Then $\Phi=\left(\Phi_{1}, \cdots, \Phi_{m}\right) \in H_{0}^{1, \alpha}(\tilde{a}, \Omega)$ and hence the following equality holds:

$$
\int_{\Omega}\left\{\nabla \Phi_{j} \cdot A_{j}+\Phi_{j} B_{j}\right\} d x=0 .
$$

Now we put $v=\left(u_{j}-k\right)^{+}$. Then $\nabla u_{i}=\nabla v$ on the set $A(k, \rho)=$ $\left\{x \in I(\rho) \mid u_{j} \geqq k\right\}$ and $\Phi_{j}=0$ outside of $A(k, \rho)$. Thus, in the set $A(k, \rho)$, we have, by (5.5),

$$
\int_{A(k, \rho)} a_{j}|\nabla v|^{\alpha} \zeta^{\alpha} d x \leqq C \int_{A(k, \rho)}\left\{\tilde{a}_{j} v^{\alpha}|\nabla \zeta|^{\alpha}+B_{j}(x) v^{\alpha} \zeta^{\alpha}+T_{j}(x)\right\} d x,
$$

where $B_{j}(x)=b_{j}^{\alpha} a_{j}^{1-\alpha}+G_{j}, \quad T_{j}(x)=\left(F_{j}+G_{j}+H_{j}^{\alpha /(\alpha-1)} \tilde{a}_{j}^{1 /(1-\alpha)}\right) \zeta^{\alpha} \in L^{p / \alpha}(I(R))$ and where $C$ is a constant depending only on $\alpha$ and $\sup \tilde{a}_{j} a_{j}^{-1}$.

We now put $\tilde{a}_{j}(\rho)=\left(\rho^{-n} \int_{I(\rho)} \tilde{a}_{j}^{p / \alpha} d x\right)^{\alpha / p}, a_{j}(\rho)=\left(\rho^{-n} \int_{I(\rho)} a_{j}^{-t} d x\right)^{1 / t}$ and $\Lambda(\rho)=\tilde{a}_{j}(\rho) a_{j}(\rho)$. Then by $(5.1), \Lambda(\rho)$ is bounded.

By the same argument as in Lemma 2.4, we obtain

$$
\begin{aligned}
\int_{A(k, \rho)} B_{j}(x) v^{\alpha} \zeta^{\alpha} d x & \leqq\left\|B_{j}\right\|_{p / \alpha}\left\{\varepsilon \left(\int_{A(k, \rho)} a_{j}|\nabla v|^{\alpha} \zeta^{\alpha} d x\right.\right. \\
+ & {\left.\left.[\text { meas } A(k, \rho)]^{1 / t} \int_{A(k, \rho)} v^{\alpha}|\nabla \zeta|^{\alpha} d x+K \int_{A(k, \rho)} v^{\alpha} \zeta^{\alpha} d x\right)\right\} . }
\end{aligned}
$$


Note that $v \leqq M\left(4 \rho_{0}\right)-k_{0}$ in $I\left(2 \rho_{0}\right)$ for $k_{0} \leqq M\left(4 \rho_{0}\right)$.

Take $\varepsilon=2^{-1}\left\|B_{j}\right\|_{p / \alpha}^{-1}$ to have

$$
\begin{aligned}
\int a_{j}|\nabla v|^{\alpha} \zeta^{\alpha} d x \leqq C\left(\rho-\rho^{\prime}\right)^{-\alpha}\left\{\left[\left(2 \rho_{0}\right)^{\alpha n / p} \tilde{a}_{j}(\rho)+\left(2 \rho_{0}\right)^{n(1 / t+\alpha / p)}\right.\right. \\
\left.\left.\left.+\left(2 \rho_{0}\right)^{\alpha+\alpha n / p}\right]\left(M\left(4 \rho_{0}\right)-k_{0}\right)^{\alpha}+\rho_{0}^{\alpha} T^{\alpha}\right\}[\text { meas } A(k, \rho)]\right)^{1-\alpha / p}
\end{aligned}
$$

for any $k_{0} \leqq M\left(4 \rho_{0}\right)$, where $T^{\alpha}=\left\|T_{j}(x)\right\|_{p / \alpha}$ and $C$ is a constant depending only on $\alpha$ and $\left\|B_{j}\right\|_{p / \alpha}$.

Let $k_{0}<k<h$. Then by Lemma 4.1 with $p=1$, (5.6) and Hölder's inequality, we have

$$
\begin{aligned}
(h-k)^{\alpha} \text { meas } A\left(k, \rho^{\prime}\right) \leqq \int_{A(k, \rho)}\left(u_{j}-k\right)^{\alpha} \zeta^{\alpha} d x & \\
\leqq & \left(\int_{A(k, \rho)}(v \zeta)^{\alpha \#} d x\right)^{\alpha / \alpha^{\#}}[\operatorname{meas} A(k, \rho)]^{\alpha / n-1 / t} \\
\leqq & \left(\left(2 \rho_{0}\right)^{n / t} a_{j}(\rho) \int_{A(k, \rho)} a_{j}|\nabla v|^{\alpha} \zeta^{\alpha} d x+\left(2 \rho_{0}\right)^{n / t} \int_{A(k, \rho)} v^{\alpha}|\nabla \zeta|^{\alpha} d x\right) \\
& \times C\left(\rho-\rho^{\prime}\right)^{-\alpha} \rho_{0}^{n(1 / t+\alpha / p)}\left\{\left(\Lambda_{j}(\rho)+\left\|a_{j}^{-1}\right\|_{t}\right)\left(M\left(4 \rho_{0}\right)-k_{0}\right)^{\alpha}+\rho_{0}^{\alpha-(n / t)} T^{\alpha}\right\} \\
& \times[\operatorname{meas} A(k, \rho)]^{\beta}
\end{aligned}
$$

for any $\alpha$ with $2 \leqq \alpha<p$, where $\beta=1+\alpha / n-1 / t-\alpha / p>1$. Thus, by Lemma 5.3 , we see that meas $A\left(k_{0}+d, \rho_{0}\right)=0$, where

$$
d=C_{0} \rho_{0}^{-1} \rho_{0}^{n / \alpha(1 / t+\alpha / p)}\left\{M\left(4 \rho_{0}\right)-k_{0}+\rho_{0}^{\theta} T\right\}\left[\text { meas } A\left(k_{0}, 2 \rho_{0}\right)\right]^{(\beta-1) / \alpha},
$$

where $\theta=1-(n / \alpha t)$ and $C_{0}$ is a constant depending only on $n, p, t, \alpha,\left\|a_{j}^{-1}\right\|_{t}$ and $\sup _{0<R} \Lambda_{j}(R)$. It is noted that $C_{0}$ is independent of $\rho_{0}$.

Hence we have

$$
M\left(\rho_{0}\right) \leqq k_{0}+C_{0}\left[\rho_{0}^{-n} \text { meas } A\left(k_{0}, 2 \rho_{0}\right)\right]^{(\beta-1) / \alpha}\left\{M\left(4 \rho_{0}\right)-k_{0}+\rho_{0}^{\theta} T\right\}
$$

for any $k_{0} \leqq M\left(4 \rho_{0}\right)$.

It is easily verified that (5.7) remains valid even when $-u_{j}$ is taken instead of $u_{j}$. In this case,

$$
M\left(\rho_{0}\right)=\sup _{I\left(\rho_{0}\right)}\left(-u_{j}\right) \text { and } A(k, \rho)=\left\{x \in I(\rho) \mid u_{j} \leqq k\right\} .
$$

Now we can assume that the inequality

$$
\text { meas } A\left(k, 2 \rho_{0}\right)<\theta_{0} \text { meas } I\left(2 \rho_{0}\right) \quad\left(0 \leqq \theta_{0}<1\right)
$$

holds for some $\theta_{0}$ and for $k=\frac{1}{2}\left(M\left(4 \rho_{0}\right)+\rho_{0}^{\theta} T\right)$. In fact, in case (5.8) is 
not valid, we may take $u_{j}^{\prime}=-u_{j}$ instead of $u_{j}$. We can therefore use Lemma 5.2 and (5.6) with the choices $\rho^{\prime}=2 \rho_{0}$ and $\rho=3 \rho_{0}$. We have, then

$$
\begin{aligned}
& (h-k)^{\alpha}\left\{\rho_{0}^{-n} \text { meas } A\left(h, 2 \rho_{0}\right)\right\}^{\alpha(n-1) / n} \\
& \quad \leqq C\left\{M\left(4 \rho_{0}\right)-k+\rho_{0}^{\theta} T\right\}^{\alpha}\left\{\rho_{0}^{-n}\left[\text { meas } A\left(k, 3 \rho_{0}\right)-\operatorname{meas} A\left(h, 3 \rho_{0}\right)\right]^{\alpha-1-1 / t}\right.
\end{aligned}
$$

for $k<h \leqq M\left(4 \rho_{0}\right)$.

Put $h_{s}\left(\rho_{0}\right)=M\left(4 \rho_{0}\right)+\rho_{0}^{\theta} T-2^{-(s+1)}\left\{\omega\left(4 \rho_{0}\right)+\rho_{0}^{\theta} T\right\}$ for $s=0,1,2, \cdots$.

We fix a natural number $N$ such that

$$
\left(C_{0} C_{1} / N\right)^{(\beta-1) / \alpha \grave{\delta}} \leqq \frac{1}{2} \quad\left(\delta=\alpha(n-1) / n\left(\alpha-1-\frac{1}{t}\right)\right)
$$

for a constant $C_{1}$ with $C 2^{r} 3^{n} \omega_{n} \leqq C_{1}$, where $\gamma=1 /(\alpha-1-1 / t)$ and $\omega_{n}$ is a volume of unit ball in $R^{n}$. First we consider it in the case when $\rho_{0}$ is a number such that $h_{s}\left(\rho_{0}\right)<M\left(4 \rho_{0}\right)$ when $s \leqq N$. Then the inequality (5.9) is valid for $h=h_{s}\left(\rho_{0}\right)$ and $k=h_{s-1}\left(\rho_{0}\right)$. Since

$$
\begin{aligned}
& h_{s}\left(\rho_{0}\right)-h_{s-1}\left(\rho_{0}\right)=2^{-(s+1)}\left\{\omega\left(4 \rho_{0}\right)+\rho_{0}^{\theta} T\right\}, \\
& M\left(4 \rho_{0}\right)+\rho_{0}^{\theta} T-h_{s-1}\left(\rho_{0}\right)=2^{-s}\left\{\omega\left(4 \rho_{0}\right)+\rho_{0}^{\theta} T\right\},
\end{aligned}
$$

(5.9) induces

$$
\begin{aligned}
& {\left[\rho_{0}^{-n} \text { meas } A\left(h_{s}\left(\rho_{0}\right), 2 \rho_{0}\right)\right]^{\delta}} \\
& \quad \leqq C 2^{r} \rho_{0}^{-n}\left[\operatorname{meas} A\left(h_{s-1}\left(\rho_{0}\right), 3 \rho_{0}\right)-\operatorname{meas} A\left(h_{s}\left(\rho_{0}\right), 3 \rho_{0}\right)\right] .
\end{aligned}
$$

Summing up each side of (5.10) for $s=1,2, \cdots, N$ we have

$$
N\left[\rho_{0}^{-n} \text { meas } A\left(h_{N}\left(\rho_{0}\right), 2 \rho_{0}\right)\right]^{\delta} \leqq C 2^{\gamma} \rho_{0}^{-n} \text { meas } A\left(h_{0}\left(\rho_{0}\right), 3 \rho_{0}\right) \leqq C_{1} .
$$

Thus we obtain

$$
C_{0}\left[\rho_{0}^{-n} \text { meas } A\left(h_{N}\left(\rho_{0}\right), 2 \rho_{0}\right)\right]^{(\beta-1) / \alpha} \leqq\left(C_{0} C_{1} / N\right)^{(\beta-1) / \alpha \grave{o}} \leqq \frac{1}{2},
$$

from which we get

$$
M\left(\rho_{0}\right) \leqq M\left(4 \rho_{0}\right)+\rho_{0}^{\theta} T-2^{-(N+2)}\left\{\omega\left(4 \rho_{0}\right)+\rho_{0}^{\theta} T\right\}
$$

if we take $k_{0}=h_{N}\left(\rho_{0}\right)$ in (5.7). Namely we obtain

$$
\omega\left(\rho_{0}\right) \leqq\left(1-2^{-(N+1)}\right)\left\{\omega\left(4 \rho_{0}\right)+\rho_{0}^{\theta} T\right\},
$$

since $m\left(\rho_{0}\right) \leqq M\left(\rho_{0}\right)$. Finally we consider it in the case when $\rho_{0}$ is a number such that $h_{N_{0}}\left(\rho_{0}\right) \geqq M\left(4 \rho_{0}\right)$ for some $N_{0}$ with $N_{0}<N$. It then follows that

$$
M\left(\rho_{0}\right) \leqq M\left(4 \rho_{0}\right) \leqq M\left(4 \rho_{0}\right)+\rho_{0}^{\theta} T-2^{-\left(N_{0}+1\right)}\left\{\omega\left(4 \rho_{0}\right)+\rho_{0}^{\theta} T\right\}
$$


and we have the inequality (5.11). Thus (5.11) is valid for any $\rho_{0}$ with $0<\rho_{0}<1$, and hence we have the inequality (5.4) using Lemma 5.4 below.

Q.E.D.

Lemma 5.4 ([9]). If there exist an $\eta$ with $0<\eta<1$ and $H>0, \theta>0$ such that

$$
\omega(\rho) \leqq \eta \omega(4 \rho)+\rho^{\theta} H \quad \text { for any } 0<\rho<1
$$

then

$$
\omega(\rho) \leqq K \rho^{\lambda}
$$

holds for some positive number $K$ and $\lambda$.

Our next attention is focused on the behavior of $u$ near the boundary $\partial \Omega$ of $\Omega$. Namely, we shall now investigate the Hölder continuity of the weak solutions of (1.1) near $\partial \Omega$.

Definition ([9]). A bounded open set $\Omega$ of $R^{n}$ is said to be $H_{0}^{1}(\Omega)$ admissible if for any $\rho<\rho_{0}$ and $x_{0} \in \partial \Omega$, and for any $v(x) \in C^{1}\left(\Omega\left(x_{0}, \rho\right)\right)$ such that $v(x)=0$ on $\partial \Omega \cap I\left(x_{0}, \rho\right)$, there exists a positive constant satisfying $\beta$

$$
|v(x)| \leqq \beta \int_{\Omega\left(x_{0}, \rho\right)} \frac{|\nabla V(t)|}{|x-t|^{n-1}} d t .
$$

Lemma 5.5 ([9]). Let $v \in H^{1,1}\left(\Omega\left(x_{0}, R\right)\right)$ with $x_{0} \in \partial \Omega$ and let $\Omega$ be $H_{0}^{1}(\Omega)$ admissible. If $v=0$ on $\partial \Omega \cap I\left(x_{0}, R\right)$, then the formula (5.2) is valid for $h>k>0$.

THEOREM 5.2. Let $u=\left(u_{1}, \cdots, u_{m}\right)$ be a weak solution of (1.1.). For some $R>0$, if $\Omega\left(x_{0}, R\right), x_{0} \in \partial \Omega$, is $H_{0}^{1}(\Omega)$-admissible and if $u=M$ on $\partial \Omega \cap I\left(x_{0}, R\right),\left(M=\left(M_{1}, \cdots, M_{m}\right) \in R\right)$, then, under the assumption (5.1) $u_{j}$ $(j=j, 2, \cdots, m)$ are Hölder continuous in $\bar{\Omega} \cap I\left(x_{0}, R\right)$.

Proof. We may assume that $u_{j}=0$ on $\partial \Omega \cap I\left(x_{0}, R\right)$. (If $u_{j} \neq 0$, we subtract the constant from $u_{j}$.) The proof is obtained in parallel with that of Theorem 5.1, where we note that

$$
\frac{1}{2}\left[M\left(4 \rho_{0}\right)+m\left(4 \rho_{0}\right)+\rho_{0}^{\theta} T\right] \geqq 0
$$

may be assumed.

Q.E.D.

In what follows, we shall assume that $\partial \Omega$ is Lipshitz continuous. Obviously $\Omega$ is $H_{0}^{1}(\Omega)$-admissible. 
If $u=\left(u_{1}, \cdots, u_{m}\right)$ is a weak solution of (1.1) and if $u_{j}-w_{j} \in H_{0}^{1, \alpha}\left(\tilde{a}_{j}, \Omega\right)$ for a Lipshitz continuous function $w_{j}, j=1,2, \cdots, m$, then $U=\left(u_{1}-w_{1}\right.$, $\left.\cdots, u_{m}-w_{m}\right) \in H_{0}^{1, \alpha}(\tilde{\alpha}, \Omega)$ and $U$ is a weak solution of the system

$$
-\operatorname{div} \tilde{A}_{j}\left(x, U, \nabla U_{j}\right)+\tilde{B}_{j}\left(x, U, \nabla U_{j}\right)=0 \quad j=1, \cdots, m,
$$

where

$$
\tilde{A}_{j}\left(x, U, \nabla U_{j}\right)=A_{j}\left(x, U+w, \nabla\left(U_{j}+w_{j}\right)\right)
$$

and

$$
\tilde{B}_{j}\left(x, U, \nabla U_{j}\right)=B_{j}\left(x, U+w, \nabla\left(U_{j}+w_{j}\right)\right), \quad\left(w=\left(w_{1}, \cdots, w_{m}\right)\right) .
$$

Moreover, we have for any $\xi \in R^{n}$

$$
\left\{\begin{array}{l}
\xi \tilde{A}_{j}(x, U, \xi) \geqq c_{0} a_{j}|\xi|^{\alpha}-\sum_{i=1}^{m} C_{i j}\left|U_{i}\right|^{\alpha}-F_{j}, \\
\left|\tilde{B}_{j}(x, U, \xi)\right| \leqq c_{1} b_{j}|\xi|^{\alpha-1}+\sum_{i=1}^{m} D_{i j}\left|U_{i}\right|^{\alpha-1}+G_{j}, \\
\left|\tilde{A}_{j}(x, U, \xi)\right| \leqq c_{2} \tilde{a}_{j}|\xi|^{\alpha-1}+\sum_{i=1}^{m} E_{i j}\left|U_{i}\right|^{\alpha-1}+H_{j},
\end{array}\right.
$$

where $c_{0}, c_{1}$ and $c_{2}$ are some constants with $0<c_{0}<1,1<c_{1}$ and $1<c_{2}$, and

$$
\begin{aligned}
& C_{i j}=c_{3}\left(c_{i j}+e_{i j}\right), \quad D_{i j}=c_{4} d_{i j}, \quad E_{i j}=c_{5} e_{i j}, \\
& F_{i j}=c_{6}\left(\left(a_{j}+\tilde{a}_{j}\right)\left|\nabla w_{\jmath}\right|^{\alpha}+\sum_{i=1}^{m}\left(c_{i j}+e_{i j}\right)\left|w_{i}\right|^{\alpha}\right)+f_{j}+\left|w_{j}\right| h_{j}, \\
& G_{i j}=c_{7}\left(b_{j}\left|\nabla w_{j}\right|^{\alpha-1}+\sum_{i=1}^{m} d_{i j}\left|w_{\imath}\right|^{\alpha-1}\right) g_{\jmath}, \\
& H_{i j}=c_{8}\left(\tilde{a}_{j}\left|\nabla w_{j}\right|^{\alpha-1}+\sum_{i=j}^{m} e_{i j}\left|w_{i}\right|^{\alpha-1}\right)+h_{j} .
\end{aligned}
$$

(Here $c_{3}, \cdots, c_{8}$ are positive constants depending only on $\alpha$.)

It is clear that $C_{i j}, D_{i j}, E_{i j}^{\alpha /(\alpha-1)} \tilde{a}_{j}^{1 /(1-\alpha)}, F_{j}, G_{j}, H_{j}^{\alpha /(\alpha-1)} \tilde{a}_{j}^{1 /(1-\alpha)} \in L^{p / \alpha}(\Omega)$. By applying Theorem 5.2, we therefore have the following

Theorem 5.3. Let $u=\left(u_{1}, \cdots, u_{m}\right)$ be a weak solution of (1.1) and let $w_{j}(j=1, \cdots, m)$ be Lipshitz continuous functions on $\Omega$. If $\partial \Omega$ is Liphistz continuous, and if $u_{j}-w_{j} \in H_{0}^{1, \alpha}\left(\tilde{a}_{j}, \Omega\right)(j=i, \cdots, m)$, then under the condition (5.1) $u_{j}$ are uniformly Hölder continuous on $\bar{\Omega}$.

\section{§6. Harnack type inequality}

In this section we shall prove the Harnack type inequality for positive weak solutions of the following system: 


$$
-\operatorname{div} A_{j}\left(x, u, \nabla u_{j}\right)+B_{j}\left(x, u, \nabla u_{j}\right)=0 \quad(j=1,2, \cdots, m),
$$

under some restrictions on the coefficients. Here each $A_{j}$ and $B_{j}$ satisfy the following three conditions (I)-(III):

(I) For any $\xi \in R^{n}$ it holds that

$$
\left\{\begin{array}{l}
\xi \cdot A_{j}(x, u, \xi) \geqq \lambda_{j} a_{j}(x)|\xi|^{\alpha}-c_{j}(x)\left|u_{j}\right|^{\alpha}-\sum_{\substack{i=1 \\
i \neq j}}^{m} c_{i j}(x)\left|u_{i}\right|^{\alpha-1}-f_{j}(x), \\
\left|B_{j}(x, u, \xi)\right| \leqq b_{j}(x)|\xi|^{\alpha-1}+\sum_{i=1}^{m} d_{i j}(x)\left|u_{i}\right|^{\alpha-1}+g_{j}(x), \\
\left|A_{j}(x, u, \xi)\right| \leqq \tilde{a}_{j}(x)|\xi|^{\alpha-1}+\sum_{i=1}^{m} e_{i j}(x)\left|u_{i}\right|^{\alpha-1}+h_{j}(x),
\end{array}\right.
$$

with some $0<\lambda_{j}<1$. The functions $a_{j}, b_{j}, c_{i j}, \cdots, h_{j}$ and $\tilde{a}_{j}$ are nonnegative and measurable. We assume that

$$
\begin{aligned}
& a_{\jmath}^{-1} \in L^{t}(\Omega) \quad \text { for any } t>1 . \\
& a_{j} \leqq a_{j}, \\
& \tilde{a}_{\jmath}, \tilde{a}_{j} a_{j}^{1-\alpha}, b_{j} a_{\jmath}^{1-\alpha}, c_{j}, c_{i j}, d_{i j}, e_{i j}^{\alpha /(\alpha-1)} \tilde{a}_{j}^{1 /(1-\alpha)}, f_{\jmath}, g_{j}, h_{j}^{\alpha /(\alpha-1)} \tilde{a}_{j}^{1 /(1-\alpha)} \in L^{p / \alpha}(\Omega),
\end{aligned}
$$

where

$$
\frac{\alpha}{p}+\frac{1}{t}<\frac{\alpha}{n} \quad \text { and } \quad(2 \leqq) \alpha<p
$$

(II) There exists another system of functions $\bar{A}_{j}\left(x, u_{j}, \nabla u_{j}\right)(j=1$, $\cdots, m$ ) such that

$$
\left\{\begin{array}{l}
\xi \cdot \bar{A}_{j}\left(x, u_{j}, \xi\right) \geqq a_{j}(x)|\xi|^{\alpha}-c_{j}(x)\left|u_{j}\right|^{\alpha}-\bar{f}_{j}(x), \\
\left|\bar{A}_{j}\left(x, u_{j}, \xi\right)\right| \leqq \tilde{a}_{j}(x)|\xi|^{\alpha-1}+e_{j}(x)\left|u_{j}\right|^{\alpha-1}+\bar{h}_{j}(x), \xi \in R^{n}
\end{array}\right.
$$

and $e_{j}^{\alpha /(\alpha-1)} \tilde{a}_{j}^{1 /(1-\alpha)}, \bar{f}_{j}, \bar{h}_{j}^{\alpha /(\alpha-1)} \tilde{a}_{j}^{1 /(1-\alpha)} \in L^{p / \alpha}(\Omega)$ with the inequalities (6.3).

(III) We assume that for any non-negative function $\Phi_{j} \in C_{0}^{\infty}(\Omega)(1 \leqq$ $j \leqq m)$ and for $u>0$ it holds that

$$
\begin{aligned}
& \int_{\Omega}\left\{\nabla \Phi_{\jmath} \cdot A_{j}\left(x, u, \nabla u_{j}\right)+\Phi_{j} B_{j}\left(x, u, \nabla u_{j}\right)\right\} d x \\
& \quad \leqq \int_{\Omega}\left\{\nabla \Phi_{j} \cdot \bar{A}_{\jmath}\left(x, u_{j}, \nabla u_{\jmath}\right)+\Phi_{j}\left(b_{j}\left|\nabla u_{j}\right|^{\alpha-1}+d_{j} u_{\jmath}^{\alpha-1}+\bar{g}_{\jmath}\right)\right\} d x
\end{aligned}
$$

with a suitable choice of $d_{j}$ and $\bar{g}_{j}$ in $L^{p / \alpha}(\Omega)$.

It is noted that the system (6.1) under the condition (I)-(III) involves general degenerate quasi-linear equations when $m=1$.

Throughout this section we consider a positive weak solution 
$u=\left(u_{1}, \cdots, u_{m}\right)$ of (6.1) under the condition (I)-(III), and we assume that $I\left(4 \rho_{0}\right) \subset \Omega$ for some positive number $\rho_{0}<1$.

Put

$$
\begin{aligned}
& \kappa_{j}=\left\|f_{j}+\bar{f}_{j}\right\|_{p / \alpha}^{1 / \alpha}+\left\|g_{j}+\bar{g}_{\jmath}\right\|_{p / \alpha}^{1 /(\alpha-1)}+\left\|\left(h_{\jmath}+\bar{h}_{j}\right)^{\alpha /(\alpha-1)} \tilde{a}_{j}^{1 /(1-\alpha)}\right\|_{p / \alpha}^{1 /(\alpha-1)}, \\
& \bar{u}_{j}=u_{j}+\kappa_{\jmath}+\varepsilon(\varepsilon>0), \bar{c}_{\jmath}=c_{j}+\left(f_{\jmath}+\bar{f}_{j}\right) / \kappa_{j}^{\alpha}, \bar{d}_{\jmath \jmath}=d_{j j}+g_{j} / \kappa_{j}^{\alpha-1}, \\
& \bar{d}_{j}=d_{j}+\bar{g}_{j} / \kappa_{j}^{\alpha-1}, \bar{e}_{j j}=e_{j \jmath}+h_{j} / \kappa_{j}^{\alpha-1}, \bar{e}_{\jmath}=e_{j}+\bar{h}_{\jmath} / \kappa_{j}^{\alpha-1}, j=1, \cdots, m .
\end{aligned}
$$

Then from (6.2) and (6.4) it follows that

$$
\left\{\begin{array}{l}
\xi \cdot A_{j}(x, u, \xi) \geqq \lambda_{\jmath} a_{j}|\xi|^{\alpha}-\bar{c}_{\jmath} \bar{u}_{j}^{\alpha}-\sum_{\substack{i=1 \\
i \neq j}}^{m} c_{i \jmath} \bar{u}_{i}^{\alpha-1}, \\
\left|B_{j}(x, u, \xi)\right| \leqq b_{\jmath}|\xi|^{\alpha+1}+\sum_{i=1}^{m} \bar{d}_{i \jmath} \bar{u}_{i}^{\alpha-1}, \\
\left|A_{j}(x, u, \xi)\right| \leqq \tilde{a}_{j}|\xi|^{\alpha-1}+\sum_{i=1}^{m} \bar{e}_{i j} \bar{u}_{i}^{\alpha-1}, \quad \xi \in R^{n},
\end{array}\right.
$$

where $\bar{d}_{i j}=d_{i j}, \bar{e}_{i j}=e_{\imath j}(i \neq j)$, and that

$$
\left\{\begin{array}{l}
\xi \cdot \bar{A}_{j}\left(x, u_{j}, \xi\right) \geqq a_{\jmath}|\xi|^{\alpha}-\bar{c}_{j} \bar{u}_{\jmath}^{\alpha}, \\
\left|\bar{A}_{j}\left(x, u_{\jmath}, \xi\right)\right| \leqq \tilde{a}_{j}|\xi|^{\alpha-1}+\bar{e}_{j} \bar{u}_{j}^{\alpha-1}, \quad \xi \in R^{n} .
\end{array}\right.
$$

We then put $p=(p / \alpha)^{\prime}=p /(p-\alpha)$,

$$
\left\{\begin{array}{l}
B_{j}(x)=\tilde{a}_{\jmath}+\tilde{a}_{\jmath} a_{i}^{1-\alpha}+b_{j} a_{\jmath}^{1-\alpha}+\bar{c}_{j}+\bar{d}_{j}+\bar{e}_{j}^{\alpha /(\alpha-1)} \tilde{a}_{\jmath}^{1 /(1-\alpha)}, \\
\tilde{B}_{\jmath}(x)=B_{j}(x)+\sum_{i=1}^{m}\left(c_{i j}+c_{j i}+\bar{d}_{i j}+\bar{d}_{j \imath}+\left(\bar{e}_{i j}^{\alpha /(\alpha-1)}+\bar{e}_{j i}^{\alpha /(\alpha-1)}\right) \tilde{a}_{j}^{1 /(1-\alpha)}\right),
\end{array}\right.
$$

and

$$
\left\{\begin{array}{l}
a_{j}(\rho)=\left(\rho^{-n} \int_{I(\rho)} a_{j}^{-t} d x\right)^{1 / t}, \quad B_{j}(\rho)=\left(\rho^{-n} \int_{I(\rho)} B_{J}^{p / \alpha} d x\right)^{\alpha / p}, \\
\tilde{B}_{j}(\rho)=\left(\rho^{-n} \int_{I(\rho)} \tilde{B}_{j}^{p / \alpha} d x\right)^{\alpha / p} .
\end{array}\right.
$$

Theorem 6.1. Let $u=\left(u_{1}, \cdots, u_{m}\right)$ be a positive weak solution of (6.1). Then

$$
\sup _{I\left(\rho_{0}\right)} \bar{u}_{\jmath} \leqq \sum_{i=1}^{m} \sup _{I\left(\rho_{0}\right)} \bar{u}_{i} \leqq C \sum_{i=1}^{m}\left(\rho_{0}^{-n} \int_{I\left(2 \rho_{0}\right)} \bar{u}_{i}^{\alpha \tilde{p}} d x\right)^{1 / \alpha \tilde{p}}
$$

where $C$ is a constant depending only on $n, p, t, \alpha, a_{j}(\rho)$ and $\tilde{B}_{j}(\rho)$.

Proof. Young's inequality with the help of (6.2) proves that the condition (1.2) is naturally satisfied by the system (6.1). Therefore, from Theorem 4.1, we have (6.8).

Q.E.D. 
Lemma 6.1. For any positive number $q_{0}>0$,

$$
\inf _{I\left(\rho_{0}\right)} \bar{u}_{j} \geqq C\left(\rho_{0}^{-n} \int_{I\left(2 \rho_{0}\right)} \bar{u}_{j}^{-q_{0}} d x\right)^{-1 / q_{0}},
$$

where $C$ is a constant depending only on $n, p, t, \alpha, a_{j}\left(2 \rho_{0}\right)$ and $B_{j}\left(2 \rho_{0}\right)$.

Proof. Take $q<0$ and $\rho^{\prime}, \rho$ such that $\rho_{0} \leqq \rho^{\prime} \leqq 2 \rho_{0}$. Put

$$
\Phi_{j}=\bar{u}_{j}^{\alpha q-\alpha+1} \zeta^{\alpha}\left(x, \rho^{\prime}, \rho\right) \text { and } \Phi_{i}=0 \text { for } i \neq j .
$$

Then, by (6.5), we have

$$
0 \leqq \int_{I(\rho)}\left\{\nabla \Phi_{j} \cdot \bar{A}_{j}\left(x, u_{j}, \nabla u_{j}\right)+\Phi_{j}\left(b_{j}\left|\nabla \bar{u}_{j}\right|^{\alpha-1}+\bar{d}_{j} \bar{u}_{j}^{\alpha-1}\right)\right\} d x .
$$

Thus we see

$$
\begin{aligned}
& -(\alpha q-\alpha+1) \int_{I(\rho)} \bar{u}_{j}^{\alpha q-\alpha \zeta \alpha} \nabla \bar{u}_{j} \cdot \bar{A}_{\jmath} d x \\
& \quad \leqq \int_{I(\rho)}\left\{\alpha \bar{u}_{j}^{\alpha q-\alpha+1} \zeta^{\alpha-1}|\nabla \zeta|\left|\bar{A}_{j}\right|+\bar{u}_{j}^{\alpha q-\alpha+1} \zeta^{\alpha}\left(b_{j}\left|\nabla \bar{u}_{j}\right|^{\alpha-1}+\bar{d}_{j} \bar{u}_{j}^{\alpha-1}\right)\right\} d x,
\end{aligned}
$$

from which, we have, by using (6.7),

$$
\int_{I(\rho)} a_{j}\left|\nabla v_{j}\right|^{\alpha} \zeta^{\alpha} d x \leqq C\left(\rho-\rho^{\prime}\right)^{-\alpha} \rho_{0}^{n}|q|^{\alpha}\left(\rho_{0}^{-n} \int_{I(\rho)} v_{\jmath}^{\alpha \tilde{p}} d x\right)^{1 / \tilde{p}},
$$

where $v_{j}=\bar{u}_{j}^{q}$ and $C$ is a constant depending only on $n, p, t, \alpha$, and $B_{j}\left(2 \rho_{0}\right)$.

Another inequalities established in Lemma 4.1 are

$$
\begin{aligned}
& \left(\left(2 \rho_{0}\right)^{-n} \int_{I\left(\rho^{\prime}\right)}\left|v_{j}\right|^{\alpha^{\#}} d x\right)^{\alpha / \alpha^{\#}} \leqq\left(\rho_{0}^{-n} \int_{I(p)}\left|v_{j} \zeta\right|^{\alpha^{\#}} d x\right)^{\alpha / \alpha \#} \\
& \leqq \\
& c_{0}\left(2 \rho_{0}\right)^{\alpha-n} a_{j}\left(2 \rho_{0}\right) \int_{I(\rho)} a_{j}\left|\nabla v_{j}\right|^{\alpha} \zeta^{\alpha} d x \\
& \quad+\left(2 \rho_{0}\right)^{\alpha}\left(\rho-\rho^{\prime}\right)^{-\alpha}\left(\left(2 \rho_{0}\right)^{-n} \int_{I(\rho)}\left|v_{j}\right|^{\alpha \tilde{p}} d x\right)^{1 / \tilde{p}} .
\end{aligned}
$$

By combining (6.10) and (6.11), we have

$$
\begin{aligned}
& \left(\rho_{0}^{-n} \int_{I\left(\rho^{\prime}\right)}\left|v_{j}\right|^{\alpha \#} d x\right)^{\alpha / \alpha^{\#}} \\
& \quad \leqq C a_{j}\left(2 \rho_{0}\right)\left(\rho-\rho^{\prime}\right)^{-\alpha} \rho_{0}^{\alpha}|q|^{\alpha}\left(\rho_{0}^{-n} \int_{I(\rho)}\left|v_{j}\right|^{\alpha \tilde{p}} d x\right)^{1 / \tilde{p}} .
\end{aligned}
$$

Put $r=\alpha^{\sharp} / \alpha, \quad q_{s}=(r / \tilde{p})^{s}\left(-q_{0}\right)$ and $\rho_{s}=\rho_{0}+\rho_{0} / 2^{s}, \quad s=0,1,2, \ldots$ With these notations the inequality (6.12) means that 


$$
\begin{aligned}
\left(\rho_{0}^{-n} \int_{I\left(\rho_{s+1}\right)} \bar{u}_{j}^{\alpha q_{s+1} \tilde{p}} d x\right)^{1 / r} & =\left(\rho_{0}^{-n} \int_{I\left(\rho_{s+1}\right)} \bar{u}_{j}^{\alpha q_{s} r} d x\right)^{1 / r} \\
& \leqq C 2^{(s+1) \alpha}\left|q_{s}\right|^{\alpha}\left(\rho_{0}^{-n} \int_{I\left(\rho_{s}\right)} \bar{u}_{j}^{\alpha q_{s} \tilde{p}} d x\right)^{1 / \tilde{p}}
\end{aligned}
$$

that is,

$$
\begin{aligned}
& \left(\rho_{0}^{-n} \int_{I\left(\rho_{s+1}\right)} \bar{u}_{j}^{\alpha q_{s+1} p} d x\right)^{1 / \alpha q_{s+1} \tilde{p}} \\
& \quad \geqq C^{1 / \alpha q_{s}}\left|q_{s}\right|^{1 / \alpha q_{s}}\left(\rho_{0}^{-n} \int_{I\left(\rho_{s}\right)} \bar{u}_{j}^{\alpha q_{s} \tilde{p}} d x\right)^{1 / \alpha q_{s} \tilde{p}} \\
& \quad \geqq C^{-\left(1 / \alpha q_{0}\right) \sum_{i=0}^{\infty}(\tilde{p} / r)^{i}}\left(\left|q_{0}\right| \frac{r}{p}\right)^{-\left(1 / q_{0}\right) \sum_{i=0}^{\infty} i(\tilde{p} / r)^{i}}\left(\rho_{0}^{-n} \int_{I\left(2 \rho_{0}\right)} \bar{u}_{j}^{-\alpha q_{0} \tilde{p}} d x\right)^{-1 / \alpha q_{0} \tilde{p}} \\
& \quad \leqq C\left(\rho_{0}^{-n} \int_{I\left(2 \rho_{0}\right)} \bar{u}_{J}^{-\alpha q_{0} \tilde{p}} d x\right)^{-1 / \alpha q_{0} \tilde{p}}
\end{aligned}
$$

Letting $s$ tend to infinity we have (6.9) for $q_{0}=q_{0} / \alpha \tilde{p}$.

LeMmA 6.2. Set $k=\left(\operatorname{meas} I\left(3 \rho_{0}\right)\right)^{-1} \int_{I(3 \rho 0)} \bar{u}_{j} d x$, and let $\tilde{u}_{j}=\bar{u}_{j} k^{-1}$ and $v_{j}=\log \tilde{u}_{j}$. Then, it follows that

$$
\left(\rho_{0}^{-n} \int_{I(3, \rho)}\left|v_{j}\right|^{\alpha \tilde{p}} d x\right)^{1 / \alpha \tilde{p}} \leqq C_{0} \quad(j=1,2, \cdots, m),
$$

where $C_{0}$ is a constant depending only on $n, p, \dot{t}, \alpha, a_{j}\left(4 \rho_{0}\right)$ and $B_{j}\left(4 \rho_{0}\right)$.

Proof. We put $\Phi_{j}=\tilde{u}_{j}^{1-\alpha} \zeta^{\alpha}\left(x, 3 \rho_{0}, 4 \rho_{0}\right)$ and $\Phi_{i}=0(i \neq j)$. Then by (6.5), we see

$$
\begin{aligned}
0 & =\int_{I\left(4 \rho_{0}\right)}\left\{\nabla \Phi_{j} \cdot A_{j}+\Phi_{j} B_{j}\right\} d x \\
& \leqq \int_{I\left(4 \rho_{0}\right)}\left\{\nabla \Phi_{j} \cdot \bar{A}_{j}+\Phi_{j}\left(b_{j}\left|\nabla \bar{u}_{j}\right|^{\alpha-1}+\bar{d}_{j} \bar{u}_{j}^{\alpha-1}\right)\right\} d x .
\end{aligned}
$$

Since $\nabla \Phi_{j}=(1-\alpha) \tilde{u}_{j}^{-\alpha} \zeta^{\alpha} \nabla \tilde{u}_{j}+\alpha \tilde{u}_{j}^{1-\alpha} \zeta^{\alpha-1} \nabla \zeta$, it follows that

$$
\begin{aligned}
& \int_{I\left(4 \rho_{0}\right)} \tilde{u}_{j}^{-\alpha \zeta \alpha} \nabla \tilde{u}_{j} \cdot \bar{A}_{j} d x \\
& \quad \leqq \int_{I\left(4 p_{0}\right)}\left\{\alpha \tilde{u}_{j}^{1-\alpha} \zeta^{\alpha-1}|\nabla \zeta|\left|\bar{A}_{j}\right|+\tilde{u}_{j}^{1-\alpha} \zeta^{\alpha}\left(b_{j}\left|\nabla \bar{u}_{j}\right|^{\alpha-1}+\bar{d}_{j} \bar{u}_{j}^{\alpha-1}\right)\right\} d x .
\end{aligned}
$$

Remind the condition (6.7) to have

$$
\begin{aligned}
& (\alpha-1) \tilde{u}_{j}^{-\alpha} \zeta^{\alpha} \nabla \tilde{u}_{j} \bar{A}_{j} \geqq(\alpha-1) k^{\alpha-1}\left(a_{j}\left|\nabla v_{j}\right|^{\alpha}-\bar{c}_{j}\right) \zeta^{\alpha}, \\
& \alpha \tilde{u}_{j}^{1-\alpha} \zeta^{\alpha-1}|\nabla \zeta|\left|\bar{A}_{j}\right| \leqq\left\{\left(\frac{\alpha-1}{4}\right) a_{j}\left|\nabla v_{j}\right|^{\alpha} \zeta^{\alpha}+\left(\frac{4}{\alpha-1}\right)^{\alpha-1} \tilde{a}_{j}^{\alpha} a_{j}^{1-\alpha}|\nabla \zeta|^{\alpha}\right. \\
& \left.+\alpha e_{j}^{\alpha /(\alpha-1)} \tilde{a}_{j}^{1 /(1-\alpha)} \zeta^{\alpha}+\alpha|\nabla \zeta|^{\alpha} \tilde{a}_{j}\right\} k^{\alpha-1}
\end{aligned}
$$


and

$$
\begin{aligned}
& \tilde{u}_{j}^{1-\alpha \zeta^{\alpha}}\left(b_{j}\left|\nabla \bar{u}_{j}\right|^{\alpha-1}+\bar{d}_{j} \bar{u}_{j}^{\alpha-1}\right) \\
& \quad \leqq\left\{\left(\frac{\alpha-1}{4}\right) a_{j}\left|\nabla v_{j}\right|^{\alpha}+\left(\frac{4}{\alpha-1}\right)^{\alpha-1}\left(b_{j} a_{j}^{1-\alpha} \zeta^{\alpha}+\bar{d}_{j} \zeta^{\alpha}\right)\right\} k^{\alpha-1} .
\end{aligned}
$$

With these estimate (6.14) becomes

$$
\int_{I(3,0)} a_{j}\left|\nabla v_{j}\right|^{\alpha} d x \leqq C \rho_{0}^{-\alpha+n} B_{j}\left(4 \rho_{0}\right) .
$$

Since (meas $\left.I\left(3 \rho_{0}\right)\right)^{-1} \int_{I\left(3 \rho_{0}\right)} v_{j} d x=0$, by Lemma 2.6, we finally have

$$
\begin{aligned}
\left(\rho_{0}^{-n} \int_{I\left(3 \rho_{0}\right)}\left|v_{j}\right|^{\alpha \tilde{p}} d x\right)^{1 / \tilde{p}} & \leqq C a_{j}\left(3 \rho_{0}\right) \rho_{0}^{n-\alpha} \int_{I\left(3 \rho_{0}\right)} a_{j}\left|\nabla v_{j}\right|^{\alpha} d x \\
& \leqq C a_{j}\left(4 \rho_{0}\right) B_{j}\left(4 \rho_{0}\right) .
\end{aligned}
$$

Lemma 6.3. Put $v_{\jmath}=\log \tilde{u}_{j}$. Then for a sufficiently small $p_{0}>0$,

$$
\rho_{0}^{-n} \int_{I\left(2 \rho_{0}\right)} e^{p_{0}\left|v_{j}\right|} d x \leqq C,
$$

where $C$ is a constant depending only on $n, p, t, \alpha, \alpha_{j}\left(3 \rho_{0}\right)$ and $B_{\jmath}\left(3 \rho_{0}\right)$.

Proof. Take $q \geqq 1$ arbitrarily. Put

$$
\begin{aligned}
& \Phi_{j}=\bar{u}_{\jmath}^{1-\alpha}\left(\left|v_{j}\right|^{\alpha q-\alpha+1}+\left[\left(\frac{\alpha}{\alpha-1}\right)(\alpha q-\alpha+1)\right]^{\alpha q-\alpha+1}\right) \zeta^{\alpha} \text { and } \Phi_{i}=0 \\
& (i \neq j) \text {, }
\end{aligned}
$$

where $\zeta=\zeta\left(x, \rho^{\prime}, \rho\right)$ with $2 \rho_{0} \leqq \rho^{\prime}<\rho \leqq 3 \rho_{0}$. Then, we see

$$
0 \leqq \int_{I(\rho)}\left\{\nabla \Phi_{j} \cdot \bar{A}_{j}+\Phi_{j}\left(b_{j}\left|\nabla \bar{u}_{j}\right|^{\alpha-1}+d_{j} \bar{u}_{j}^{\alpha-1}\right)\right\} d x .
$$

The exact form of $\nabla \Phi_{j}$ is given by

$$
\begin{aligned}
\nabla \Phi_{j}= & \left\{( 1 - \alpha ) \left(\left|v_{j}\right|^{\alpha q-\alpha+1}+\left[\frac{\alpha}{\alpha-1}(\alpha q-\alpha+1)\right]^{\alpha q-\alpha+1}\right.\right. \\
& \left.\left.+(\alpha q-\alpha+1)\left|v_{j}\right|^{\alpha q-\alpha} \operatorname{sign} v_{j}\right)\right\} \bar{u}_{j}^{-\alpha} \zeta^{\alpha} \nabla \bar{u}_{J} \\
& +\alpha\left(\left|v_{j}\right|^{\alpha q-\alpha+1}+\left[\frac{\alpha}{\alpha-1}(\alpha q-\alpha+1)\right]^{\alpha q-\alpha+1}\right) \bar{u}_{j}^{1-\alpha} \zeta^{\alpha-1} \nabla \zeta .
\end{aligned}
$$

While, the next inequality comes from Young's inequality

$$
\begin{aligned}
& (\alpha q-\alpha+1)\left|v_{j}\right|^{\alpha q-\alpha} \\
& \quad \leqq\left(\frac{\alpha-1}{\alpha}\right)\left(\left|v_{j}\right|^{\alpha q-\alpha+1}+\left[\frac{\alpha}{\alpha-1}(\alpha q-\alpha+1)\right]^{\alpha q-\alpha+1}\right)
\end{aligned}
$$


Thus we see that

$$
\begin{gathered}
(1-\alpha)\left(\left|v_{j}\right|^{\alpha q-\alpha+1}+\left[\frac{\alpha}{\alpha-1}(\alpha q-\alpha+1)\right]^{\alpha q-\alpha+1}\right) \\
+(\alpha q-\alpha+1)\left|v_{j}\right|^{\alpha q-\alpha} \operatorname{sign} v_{j} \\
\leqq-\frac{(\alpha-1)^{2}}{\alpha}\left(\left|v_{j}\right|^{\alpha q-\alpha+1}+\left[\frac{\alpha}{\alpha-1}(\alpha q-\alpha+1)\right]^{\alpha q-\alpha+1}\right)<0 .
\end{gathered}
$$

Therefore, by the structure (6.7), we have

$$
\begin{aligned}
& \frac{(\alpha-1)^{2}}{\alpha} \int_{I(\rho)}\left(\left|v_{j}\right|^{\alpha q-\alpha+1}+\left[\frac{\alpha}{\alpha-1}(\alpha q-\alpha+1)\right]^{\alpha q-\alpha+1}\right) a_{j}\left|v_{j}\right| \zeta^{\alpha} d x \\
& \leqq C \int_{I(\rho)}\left(\left|v_{j}\right|^{\alpha q-\alpha+1}+\left[\frac{\alpha}{\alpha-1}(\alpha q-\alpha+1)\right]^{\alpha q-\alpha+1}\right) \\
& \times\left\{\bar{u}_{j}^{-\alpha} \zeta^{\alpha} \bar{c}_{j} \bar{u}_{j}^{\alpha}+\alpha \bar{u}_{j}^{1-\alpha \zeta^{\alpha-1}}|\nabla \zeta|\left(\tilde{a}_{j}\left|\nabla \bar{u}_{j}\right|^{\alpha-1}+\bar{e}_{j} \bar{u}_{j}^{\alpha-1}\right)\right. \\
& \left.+\bar{u}_{j}^{1-\alpha \zeta^{\alpha}}\left(b_{j}\left|\nabla \bar{u}_{j}\right|^{\alpha-1}+\bar{d}_{j} \bar{u}_{j}^{\alpha+1}\right)\right\} d x
\end{aligned}
$$

By an obvious equality $\bar{u}_{j}^{-\alpha}\left|\nabla \bar{u}_{j}\right|=\left|\nabla v_{j}\right|$ we can see the right-hand side of (6.17)

$$
\begin{array}{r}
=C \int_{I(\rho)}\left(\left|v_{j}\right|^{\alpha q-\alpha+1}+\left[\frac{\alpha}{\alpha-1}(\alpha q-\alpha+1)\right]^{\alpha q-\alpha+1}\right)\left\{\alpha \tilde{a}_{j}\left|\nabla v_{j}\right|^{\alpha-1} \zeta^{\alpha-1}|\nabla \zeta|\right. \\
\left.+b_{j}\left|\nabla v_{j}\right|^{\alpha-1} \zeta^{\alpha}+\bar{c}_{j} \zeta^{\alpha}+\bar{d}_{j} \zeta^{\alpha}+\bar{e}_{j} \zeta^{\alpha-1}|\nabla \zeta|\right\} d x \\
\leqq C \int_{I(\rho)}\left(\left|v_{j}\right|^{\alpha q-\alpha+1}+\left[\frac{\alpha}{\alpha-1}(\alpha q-\alpha+1)\right]^{\alpha q-\alpha+1}\right) \\
\quad \times\left(\frac{1}{2 C} \frac{(\alpha-1)^{2}}{\alpha} a_{j}\left|\nabla v_{j}\right|^{\alpha \zeta^{\alpha}}+|\nabla \zeta|^{\alpha} B_{j}(x)\right) d x .
\end{array}
$$

Then, we have by (6.17), using (6.16) again,

$$
\begin{aligned}
& \int_{I(\rho)} a_{j}\left|v_{j}\right|^{\alpha q-\alpha}\left|\nabla v_{j}\right|^{\alpha} \zeta^{\alpha} d x \\
& \quad \leqq(\alpha q-\alpha+1)^{-1} C \int_{I(\rho)}\left(\left|v_{j}\right|^{\alpha q}+\left[\frac{\alpha}{\alpha-1}(\alpha q-\alpha+1)\right]^{\alpha q-\alpha+1}\right)|\nabla \zeta|^{\alpha} B_{j}(x) d x .
\end{aligned}
$$

Here we have used the fact that

$$
\left|v_{j}\right|^{\alpha q-\alpha+1} \leqq\left|v_{j}\right|^{\alpha q}+\left[\frac{\alpha}{\alpha-1}(\alpha q-\alpha+1)\right]^{\alpha q-\alpha+1} .
$$

Put $V_{j}=v_{j}^{q}$. Then, by an obvious equality $\left|\nabla V_{j}\right|^{\alpha}=q^{\alpha}\left|v_{j}\right|^{\alpha q-\alpha}$, we have

$$
\begin{aligned}
\int_{I(\rho)} a_{j}\left|\nabla V_{j}\right|^{\alpha} \zeta^{\alpha} d x & \leqq C \int_{I(\rho)}\left(\left|V_{\jmath}\right|^{\alpha}+(\gamma q)^{\alpha q}\right)|\nabla \zeta| B_{j}(x) d x \\
& \leqq C\left(\rho-\rho^{\prime}\right)^{-\alpha} \rho_{0}^{-n}\left\{\left(\rho_{0}^{-n} \int_{I(\rho)}\left|V_{j}\right|^{\alpha p} d x\right)^{1 / p}+(\gamma q)^{\alpha q}\right\},
\end{aligned}
$$


where $C$ is a constant depending only on $n, p, t, \alpha$ and $B_{j}\left(3 \rho_{0}\right)$, and $\gamma$ depends on $\alpha$.

This results and Lemma 4.1 guarantee the following

$$
\left(\rho_{0}^{-n} \int_{I\left(\rho^{\prime}\right)}\left|V_{j}\right|^{\alpha^{\sharp}} d x\right)^{\alpha / \alpha \#} \leqq C\left(\rho-\rho^{\prime}\right)^{-\alpha} \rho_{0}^{\alpha}\left\{\left(\rho_{0}^{-n} \int_{I(\rho)}\left|V_{j}\right|^{\alpha \tilde{p}} d x\right)^{1 / \tilde{p}}+(\gamma q)^{\alpha q}\right\},
$$

where $C$ depends only on $n, p, t, \alpha, a_{j}\left(3 \rho_{0}\right)$ and $B_{j}\left(3 \rho_{0}\right)$.

Putting $q_{s}=(r / p)^{s}\left(r=\alpha^{\sharp} / \alpha\right)$ and $\rho_{s}=2 \rho_{0}+2^{-s} \rho_{0}, s=0,1, \cdots$, the above inequality proves

$$
\begin{aligned}
& \left(\rho_{0}^{-n} \int_{I\left(\rho_{s+1}\right)}\left|v_{j}\right|^{\alpha q_{s+1} \tilde{p}} d x\right)^{1 / \alpha q_{s+1} \tilde{p}} \\
& \quad \leqq C^{1 / \alpha q_{s} 2^{s / \alpha q_{s}}}\left\{\left(\rho_{0}^{-n} \int_{I\left(\rho_{s}\right)}\left|v_{j}\right|^{\alpha q_{s} \tilde{p}} d x\right)^{1 / \alpha q_{s} \tilde{p}}+\gamma q_{s}\right\} \\
& \quad \leqq C\left\{\left(\rho_{0}^{-n} \int_{I\left(\rho_{s}\right)}\left|v_{j}\right|^{\alpha q_{s} \tilde{p}} d x\right)^{1 / \alpha q_{s} p}+\sum_{\ell=0}^{s} \gamma q_{\ell}\right\}
\end{aligned}
$$

that is, for any positive number $q \geqq 1$

$$
\left(\rho_{0}^{-n} \int_{I(2 \rho 0)}\left|v_{j}\right|^{q} d x\right)^{1 / q} \leqq C\left\{\left(\rho_{0}^{-n} \int_{I(3 \rho 0)}\left|v_{j}\right|^{\alpha \tilde{p}} d x\right)^{1 / \alpha \tilde{p}}+q\right\} \leqq C\left\{C_{0}+q\right\}
$$

from which we have (6.15).

Q.E.D.

Lemma 6.4. Let $u=\left(u_{1}, \cdots, u_{m}\right)$ be a positive solution of (6.1). Then

$$
\inf _{I\left(2 \rho_{0}\right)} \bar{u}_{j} \geqq C_{1}
$$

where $C_{1}$ is a positive constant depending only on $n, p, t, \alpha, a_{j}\left(3 \rho_{0}\right)$ and $B_{j}\left(3 \rho_{0}\right)$.

Proof. Put $v_{j}=\log \left\{\min \left(1, \tilde{u}_{j}\right)\right\}$ and take $\Phi_{j}=\bar{u}_{j}^{1-\alpha}\left|v_{j}\right|^{\alpha q-\alpha+1} \zeta^{\alpha}$ with $q \leqq 1$, where $\zeta=\zeta\left(x, \rho^{\prime}, \rho\right)$ with $2 \rho_{0} \leqq \rho^{\prime}<\rho \leqq 3 \rho_{0}$. Then, we have

$$
\begin{aligned}
\nabla \Phi_{j}=\left((1-\alpha)\left|v_{j}\right|^{\alpha q-\alpha+1}-(\alpha q-\alpha+1)\left|v_{j}\right|^{\alpha q-\alpha}\right) \bar{u}_{j}^{-\alpha} \zeta^{\alpha} \nabla \bar{u}_{j} \\
+\alpha \bar{u}_{j}^{1-\alpha}\left|v_{j}\right|^{\alpha q-\alpha+1} \zeta^{\alpha-1}|\nabla \zeta| .
\end{aligned}
$$

Thus, by (6.5) and the structure (6.7),

$$
\begin{aligned}
(\alpha q- & \alpha+1) \int_{I(\rho)} a_{\jmath}\left|v_{j}\right|^{\alpha q-\alpha}\left|\nabla v_{j}\right|^{\alpha \alpha} \zeta^{\alpha} d x \\
\leqq & \int_{\Omega}\left\{\alpha \bar{u}_{j}^{1-\alpha}\left|v_{j}\right|^{\alpha q-\alpha+1} \zeta^{\alpha-1}|\nabla \zeta|\left(\tilde{a}_{j}\left|\nabla \bar{u}_{\jmath}\right|^{\alpha-1}+\bar{e}_{j} \bar{u}_{\jmath}^{\alpha-1}\right)\right. \\
& \quad+\bar{u}_{j}^{1-\alpha}\left|v_{\jmath}\right|^{\alpha q-\alpha+1} \zeta^{\alpha}\left(b_{j}\left|\nabla \bar{u}_{\jmath}\right|^{\alpha-1}+\bar{d}_{\jmath} \bar{u}_{j}^{\alpha-1}\right) \\
& \left.\quad+\left((\alpha-1)\left|v_{j}\right|^{\alpha q-\alpha+1}+(\alpha q-\alpha+1)\left|v_{j}\right|^{\alpha q-\alpha}\right) \bar{u}_{\jmath}^{-\alpha} \zeta^{\alpha} \bar{c}_{\jmath} \bar{u}_{j}^{\alpha}\right\} d x
\end{aligned}
$$


Since $\left|v_{j}\right|^{\alpha q-\alpha+1} \leqq\left|v_{j}\right|^{\alpha q}+(1 /(\alpha q-\alpha+1))$ and $\left|v_{j}\right|^{\alpha q-\alpha} \leqq\left|v_{j}\right|^{\alpha q}+1 / q$ hold, we have

$$
\int_{I(\rho)} a_{j}\left|\nabla\left(v_{j}^{q}\right)\right|^{\alpha} \zeta^{\alpha} d x \leqq C q^{\alpha}\left(\rho-\rho^{\prime}\right)^{-\alpha} \rho_{0}^{n}\left\{\left(\rho_{0}^{-n} \int_{I(\rho)}\left|v_{j}\right|^{\alpha q \tilde{p}} d x\right)^{1 / \tilde{p}}+\frac{1}{q}\right\},
$$

where $C$ is a constant depending only on $n, p, t, \alpha, a_{j}\left(3 \rho_{0}\right)$ and $B_{j}\left(3 \rho_{0}\right)$. From which we have

$$
\sup _{I(2 \rho 0)}\left|v_{j}\right| \leqq C\left\{C_{0}+\gamma\right\}
$$

in a similar manner to the case of Theorem 4.1, that is, (6.18) holds.

Q.E.D.

We are now ready to state the Harnack theorem.

Theorem 6.2. Let $u=\left(u_{1}, \cdots, u_{m}\right)$ be a positive weak solution of (6.1) under the condition (I)-(III). Then

$$
\sum_{j=1}^{m} \sup _{I\left(\rho_{0}\right)} u_{j} \leqq C \sum_{j=1}^{m}\left(\inf _{I\left(\rho_{0}\right)} u_{j}+\kappa_{j}\right)
$$

where $C$ is a positive constant depending only on $n, p, t, \alpha, a_{j}\left(4 \rho_{0}\right)$ and $B_{j}\left(4 \rho_{0}\right)$ $(j=1,2, \cdots, m)$.

Proof. From (6.15), we have

$$
\left(\rho_{0}^{-n} \int_{I\left(2 \rho_{0}\right)} \bar{u}_{\jmath}^{p_{0}} d x\right)^{1 / \rho_{0}} \leqq C\left(\rho_{0}^{-n} \int_{I\left(2_{0}\right)} \bar{u}_{j}^{-p_{0}} d x\right)^{-1 / p_{0}} .
$$

Combining this inequality with (6.9), we obtain

$$
\left(\rho_{0}^{-n} \int_{I\left(2 \rho_{0}\right)} \bar{u}_{j}^{p_{0}} d x\right)^{1 / p_{0}} \leqq C \inf _{I\left(\rho_{0}\right)} \bar{u}_{j}, \quad j=1,2, \cdots, m .
$$

Hence Theorem 6.2 can be proved if we show

$$
\sum_{j=1}^{m} \sup _{I\left(\rho_{0}\right)} \bar{u}_{j} \leqq C \sum_{j=1}^{m}\left(\rho_{0}^{-n} \int_{I\left(2 \rho_{0}\right)} \bar{u}_{j}^{p_{0}} d x\right)^{1 / p_{0}}
$$

In fact this is true as is shown below. Put $\Phi_{\jmath}=\bar{u}_{j}^{\alpha q-\alpha+1 \zeta^{\alpha}}$ with $q>0$, $q \neq(\alpha-1) / \alpha$, where $\zeta=\zeta\left(x, \rho^{\prime}, \rho\right)$ with $\rho_{0} \leqq \rho^{\prime}<\rho \leqq 2 \rho_{0}$.

In case $0<q<(\alpha-1) / \alpha, \Phi_{i}(i \neq j)$ is defined to be 0 , and

$$
0 \leqq \int_{I(\rho)}\left\{\nabla \Phi_{j} \cdot \bar{A}_{j}+\Phi_{j}\left(b_{j}\left|\nabla \bar{u}_{j}\right|^{\alpha-1}+\bar{d}_{j} \bar{u}_{j}^{\alpha-1}\right)\right\} d x
$$

is shown by (6.5). Since $\nabla \Phi_{j}=(\alpha q-\alpha+1) \bar{u}_{j}^{\alpha q-\alpha \zeta^{\alpha}} \nabla \bar{u}_{j}+\alpha \bar{u}_{j}^{\alpha q-\alpha+1} \zeta^{\alpha-1} \nabla \zeta$, by 
(6.7), we have

$$
\begin{aligned}
& -(\alpha q-\alpha+1) \int_{I(\rho)} a_{j} \bar{u}_{j}^{\alpha q-\alpha}\left|\nabla \bar{u}_{j}\right|^{\alpha} \zeta^{\alpha} d x \\
& \leqq \int_{I(\rho)}\left\{\alpha \bar{u}_{j}^{\alpha q-\alpha+1} \zeta^{\alpha-1}|\nabla \zeta|\left(\tilde{a}_{j}\left|\nabla \bar{u}_{j}\right|^{\alpha-1}+\bar{e}_{j} \bar{u}_{j}^{\alpha-1}\right)\right. \\
& \left.\quad+\bar{u}_{j}^{\alpha q-\alpha+1} \zeta^{\alpha}\left(b_{j}\left|\nabla \bar{u}_{j}\right|^{\alpha-1}+\bar{d}_{j} \bar{u}_{j}^{\alpha-1}\right)+|\alpha q-\alpha+1| \bar{c}_{j} \bar{u}_{j}^{\alpha q}\right\} d x .
\end{aligned}
$$

Put $v_{j}=\bar{u}_{j}^{q}$. Then, by the same argument as before, we have

$$
\int_{I(\rho)} a_{j}\left|\nabla v_{j}\right|^{\alpha} \zeta^{\alpha} d x \leqq C_{q} \int_{I(\rho)}|\nabla \zeta|^{\alpha} B_{j}(x) v_{j}^{\alpha} d x
$$

Therefore,

$$
\sum_{j=1}^{m} \int_{I(\rho)} a_{j}\left|\nabla v_{j}\right|^{\alpha} \zeta^{\alpha} d x \leqq C_{q}\left(\rho-\rho^{\prime}\right)^{-\alpha} \rho_{0}^{n}\left(\rho_{0}^{-n} \int_{I(\rho)}\left(\sum_{j=1}^{m} v_{j}\right)^{\alpha \tilde{p}} d x\right)^{1 / \tilde{p}}
$$

where $C_{q}$ is a constant depending only on $n, p, q, t, \alpha, a_{j}\left(2 \rho^{0}\right)$ and $B_{j}\left(2 \rho_{0}\right)$ $(j=1,2, \cdots, m)$.

In case $(\alpha-1) / \alpha<q$, we define $\Phi_{j}=\bar{u}_{j}^{\alpha q-\alpha+1} \zeta^{\alpha}(j=1,2, \cdots, m)$ to have

$$
\sum_{j=1}^{m} \int_{I(\rho)}\left\{\nabla \Phi_{j} \cdot A_{j}+\Phi_{j} B_{j}\right\} d x=0
$$

Since $\nabla \Phi_{j}=(\alpha q-\alpha+1) \bar{u}_{j}^{\alpha q-\alpha} \zeta^{\alpha} \nabla u_{j}+\alpha \bar{u}_{j}^{\alpha q-\alpha+1} \zeta^{\alpha-1} \nabla \zeta$,

$$
\begin{aligned}
(\alpha q-\alpha+1) \sum_{j=1}^{m} \lambda_{j} \int_{I(\rho)} a_{j} u_{j}^{\alpha q-\alpha}\left|\nabla \bar{u}_{j}\right|^{\alpha} \zeta^{\alpha} d x \\
\leqq \sum_{j=1}^{m} \int_{I(\rho)}\left\{\alpha \bar{u}_{j}^{\alpha q-\alpha+1} \zeta^{\alpha-1}|\nabla \zeta|\left(\tilde{a}_{j}\left|\nabla \bar{u}_{j}\right|^{\alpha-1}+\sum_{i=1}^{m} \bar{e}_{i j} \bar{u}_{i}^{\alpha-1}\right)\right. \\
+\bar{u}_{j}^{\alpha q-\alpha+1} \zeta^{\alpha}\left(b_{j}\left|\nabla \bar{u}_{j}\right|^{\alpha-1}+\sum_{i=1}^{m} \bar{d}_{i j} \bar{u}_{i}^{\alpha-1}\right) \\
\left.+(\alpha q-\alpha+1) \zeta^{\alpha}\left(\bar{c}_{j} \bar{u}_{j}^{\alpha q}+\sum_{\substack{i=1 \\
i \neq j}}^{m} c_{i j} \bar{u}_{i}^{\alpha-1} \bar{u}_{j}^{\alpha q-\alpha}\right)\right\} d x
\end{aligned}
$$

is proved by using the condition (6.6). By Lemma 6.4, $\bar{u}_{j}^{\alpha q-\alpha} \leqq C_{1} \bar{u}_{j}^{\alpha q-\alpha+1}$ is true. Noting $\alpha q /(\alpha-1) \geqq 1$, we see, by using Young's inequality,

$$
\bar{u}_{i}^{\alpha-1} \bar{u}_{j}^{\alpha-\alpha+1} \leqq \bar{u}_{i}^{\alpha q}+\bar{u}_{j}^{\alpha q} \quad \text { and } \quad \bar{u}_{i}^{\alpha-1} \bar{u}_{j}^{\alpha q-\alpha} \leqq C_{1}^{-1}\left(\bar{u}_{i}^{\alpha q}+\bar{u}_{j}^{\alpha q}\right)
$$

Thus, putting $v_{j}=\bar{u}_{j}^{q}(j=1,2, \cdots, m)$, we have

$$
\sum_{j=1}^{m} \int_{I(\rho)} a_{j}\left|\nabla v_{j}\right|^{\alpha} \zeta^{\alpha} d x \leqq C_{q}\left(\rho-\rho^{\prime}\right)^{-\alpha} \rho^{n} \sum_{j=1}^{m}\left(\rho_{0}^{-n} \int_{I(\rho)}\left(\sum_{j=1}^{m} v_{j}\right)^{\alpha \tilde{p}} d x\right)^{1 / \tilde{p}}
$$


where $C_{q}$ is a constant depending only on $n, p, q, t, \alpha, a_{j}\left(2 \rho_{0}\right)$ and $B_{j}\left(2 \rho_{0}\right)$ $(j=1,2, \cdots, m)$.

We have therefore obtained (6.21) for any $q>0$ with $q \neq(\alpha-1) / \alpha$.

Let $q_{0}$ be a sufficiently small number such that $0<\alpha q_{0} \tilde{p} \leqq p_{0}$ and $q_{0}(r / p)^{s} \neq(\alpha-1) / \alpha, s=0,1,2, \cdots$. Then, (6.20) is obtained in a similar manner to the case of Theorem 4.1, which was to be proved. Q.E.D.

To close this section we give an illustrative example.

Take a vector function

$$
C_{j}(x, u)=\left(\sum_{\substack{i=1 \\ \imath \neq j}}^{m} c_{\imath j}^{1} u_{\imath}^{(\alpha-1)^{2 / \alpha}} \operatorname{sign} \frac{\partial u_{i}}{\partial x_{1}}, \cdots, \sum_{\substack{i=1 \\ i \neq j}}^{m} c_{i j}^{n} u_{i}^{(\alpha-1)^{2 / \alpha}} \operatorname{sign} \frac{\partial u_{i}}{\partial x_{n}}\right) .
$$

Let $\bar{A}_{i}\left(x, u_{j}, \nabla u_{j}\right)=\left(A_{1 j}, \cdots, A_{n j}\right)$ and a scalar function $\bar{B}_{j}\left(x, u, \nabla u_{j}\right)$ be given and satisfy the following inequalities

$$
\left\{\begin{array}{l}
\xi \cdot \bar{A}_{j}\left(x, u_{j}, \xi\right) \geqq a_{j}|\xi|^{\alpha}-c_{j}\left|u_{j}\right|^{\alpha}-\bar{f}_{j}, \\
\left|\bar{B}_{j}(x, u, \xi)\right| \leqq b_{j}|\xi|^{\alpha-1}+\sum_{i=1}^{m} d_{i j}\left|u_{i}\right|^{\alpha-1}+g_{j}, \\
\left|A_{j}\left(x, u_{j}, \xi\right)\right| \leqq \tilde{a}_{j}|\xi|^{\alpha-1}+e_{j}\left|u_{j}\right|^{\alpha-1}+\bar{h}_{j}, \quad \xi \in R^{n}
\end{array}\right.
$$

$j=1, \cdots, m$. Here $a_{j}, b_{j}, \cdots, \bar{h}_{j}$ and $\tilde{a}_{j}$ are non-negative measurable functions in $\Omega, a_{j}^{-1} \in L^{t}(\Omega)$ for any $t>1, a_{j} \leqq \tilde{a}_{j}$, and $\tilde{a}_{j}, \tilde{a}_{j}^{\alpha} a_{\jmath}^{1-\alpha}, b_{j} a_{j}^{1-\alpha}, c_{j}, d_{i_{j}}$, $\bar{f}_{j}, g_{j},\left(c_{i j}^{k}\right)^{\alpha /(\alpha-1)} a_{j}^{1 /(1-\alpha)}, e_{j}^{\alpha /(\alpha-1)} \tilde{a}_{j}^{1 /(1-\alpha)}, \bar{h}_{j}^{\alpha /(\alpha-1)} \tilde{a}_{j}^{1 /(1-\alpha)} \in L^{p / \alpha}(\Omega)$, and $c_{i j} \in H^{1, p / \alpha}(\Omega)$.

Define $A_{j}\left(x, u, \nabla u_{j}\right)=A_{j}\left(x, u_{j}, \nabla u_{j}\right)+C_{j}(x, u)$ and

$$
B_{j}\left(x, u, \nabla u_{\jmath}\right)=\bar{B}_{j}\left(x, u, \nabla u_{\jmath}\right)+\sum_{i=1}^{m} \tilde{d}_{i j} u_{i}^{\alpha-1}+\sum_{\substack{i=1 \\ \imath \neq j}}^{m} \tilde{c}_{i j} u_{i}^{(\alpha-1) 2 / \alpha},
$$

for some choices of functions, $\tilde{d}_{i j}, \tilde{c}_{i j} \in L^{p / \alpha}(\Omega)$.

We assume that for any $i(i \neq j)$

$$
\sum_{k=1}^{n}\left|\frac{c_{i j}^{k}}{x_{k}}\right|+\tilde{c}_{i j} \leqq 0 \quad \text { and } \quad d_{i j}+\tilde{d}_{i j} \leqq 0
$$

Then, the system

$$
-\operatorname{div} A_{j}\left(x, u, \nabla u_{\jmath}\right)+B_{j}\left(x, u, \nabla u_{j}\right)=0 \quad(j=1,2, \cdots, m)
$$

satisfies the condition (I)-(III).

\section{REFERENCES}

[1] F. Mandras, Il problema di Dirichlet per una classe di sistemi lineari non-uniformente ellittici, Rend. Sem. Fac. Sci. Univ. Cagliari, 45 (1975), 333-345. 
[2] F. Mandras, Principio di massimo debole per sotto-soluzioni di sistemi lineari ellittici debolmente accoppiati, Boll. Un. Mat. Ital. (5) 13-A (1976), 592-600.

[3] - Disegualianza di Harnack per sistemi ellittici debolmente accoppiati, Boll. Un. Mat. Ital. (5) 14-A (1977), 313-321.

[ 4 ] J. Moser, On Harnack's theorem for elliptic differential equations, Comm. Pure. Appl. Math., 14 (1961), 577-591.

[5] M. K. V. Murthy and G. Stampacchia, Boundary value problems for some degenerate-elliptic operators, Ann. Pura. Appl., 80 (1968), 1-122.

[6] L. Nirenberg, On elliptic differential equations, Ann. Scula Norm. Sup. Pisa, 13 (1959), 116-162.

[7] J. Serrin, Local behavior of solutions of quasi-linear equations, Acta Math., 111 (1964), 247-302.

[8] G. Stampacchia, Équations elliptiques du second ordre à coéfficients discontinus, Séminaire sur les équations aux dérivées partielles, Collège de France (mai 1964).

[ 9 ] — Le problème de Dirichlet pour les équations discontinus, Ann. Inst. Fourier, 15 (1965) 189-258.

[10] N. S. Trudinger, On Harnack type inequalities and their application to quasilinear elliptic equations, Comm. Pure. Appl. Math., 20 (1967), 721-747. MR 37 \#1788.

[11] N. S. Trudinger, Linear elliptic operator with measurable coefficients, Ann. Scuola Norm. Sup. Pisa, 27 (1973) 265-308.

Department of Mathematics

Aichi University of Education

Kariya, 448 Japan 